\title{
Conventional and unconventional mechanisms for capping viral mRNA
}

Etienne Decroly' ${ }^{1}$ François Ferron ${ }^{1}$, Julien Lescar ${ }^{1,2}$ and Bruno Canard ${ }^{1}$

Abstract | In the eukaryotic cell, capping of mRNA $5^{\prime}$ ends is an essential structural modification that allows efficient mRNA translation, directs pre-mRNA splicing and mRNA export from the nucleus, limits mRNA degradation by cellular 5' $-3^{\prime}$ exonucleases and allows recognition of foreign RNAs (including viral transcripts) as 'non-self'. However, viruses have evolved mechanisms to protect their RNA 5' ends with either a covalently attached peptide or a cap moiety (7-methyl-Gppp, in which p is a phosphate group) that is indistinguishable from cellular mRNA cap structures. Viral RNA caps can be stolen from cellular mRNAs or synthesized using either a host- or virus-encoded capping apparatus, and these capping assemblies exhibit a wide diversity in organization, structure and mechanism. Here, we review the strategies used by viruses of eukaryotic cells to produce functional mRNA $5^{\prime}$-caps and escape innate immunity.

Pre-mRNA splicing A post-transcriptional modification of pre-mRNA, in which introns are excised and exons are joined in order to form a translationally functional, mature mRNA.
'Centre National de la Recherche Scientifique and Aix-Marseille Université, UMR 6098, Architecture et Fonction des Macromolécules Biologiques, 163 avenue de Luminy, 13288 Marseille cedex 09, France.

${ }^{2}$ School of Biological Sciences, Nanyang Technological University, 60 Nanyang Drive, Singapore 637551 , Republic of Singapore. Correspondence to B.C. e-mail:Bruno.Canard@ afmb.univ-mrs.fr

doi:10.1038/nrmicro2675 Published online

5 December 2011
The cap structure found at the $5^{\prime}$ end of eukaryotic mRNAs consists of a 7 -methylguanosine $\left(\mathrm{m}^{7} \mathrm{G}\right)$ moiety linked to the first nucleotide of the transcript via a $5^{\prime}-5^{\prime}$ triphosphate bridge ${ }^{1}$ (FIG. 1a). The cap has several important biological roles, such as protecting mRNA from degradation by $5^{\prime}$ exoribonucleases and directing ' pre-mRNA splicing and mRNA export from the nucleus ${ }^{2}$. In addition, the cap confers stability to mRNAs and ensures their efficient recognition by eukaryotic translation initiation factor 4E (eIF4E) for translation ${ }^{3,4}$. Conversely, RNA molecules with unprotected $5^{\prime}$ ends are degraded in cytoplasmic granular compartments called processing bodies (P-bodies) $)^{5}$. Uncapped RNAs, such as nascent viral transcripts, may also be detected as 'non-self' by the host cell, triggering (in mammalian cells) an antiviral innate immune response through the production of interferons ${ }^{6,7}$.

During virus-host co-evolution, viral RNA-capping pathways that lead to the same cap structure as that of host mRNAs have been selected for as efficient mechanisms to ensure both escape from detection by the innate immune system and efficient production of viral proteins. Compared with canonical eukaryotic mRNA capping, viral mRNA capping is highly diverse in terms of its genetic components, protein domain organization, enzyme structures and reaction mechanisms. Here, we review what is known about the various mRNA-capping pathways used by viruses that infect eukaryotes, paying particular attention to human pathogens. We also attempt to connect the pathways, machineries, structures and reactions involved in the viral RNA-capping process, and the specific cellular factors that trigger a response from the innate immune system.

\section{Capping, decapping and turnover of host RNA}

Nascent cellular mRNAs are generally produced in the nucleus in a $5^{\prime}$-triphosphate form and are modified cotranscriptionally by a set of cap-synthesizing enzymes. These enzymes are recruited by the DNA-dependent RNA polymerase RNA pol II on pausing, once the transcript is approximately $20-25$ nucleotides long. Interestingly, two viruses - vaccinia virus (from the Poxviridae family of double-stranded DNA (dsDNA) viruses) and mammalian orthoreovirus (from the dsRNA Reoviridae family) - played a major part in the discovery of the RNA cap ${ }^{1,8-11}$, because they produce high levels of capped viral mRNAs and encode their own capping machinery, allowing bona fide RNA cap synthesis in vitro. Mammalian orthoreovirus mRNAs and dsRNA genomes were first shown to be blocked at the $5^{\prime}$ end, preventing phosphorylation by polynucleotide kinase ${ }^{9,10}$. Purified mammalian orthoreovirus preparations were then demonstrated to be able to methylate mRNA $5^{\prime}$ ends, and the full mRNA cap structures were deciphered for both dsRNA orthoreoviruses ${ }^{9,10,12}$ (those targeting humans and insects) and the dsDNA species vaccinia virus ${ }^{11}$, followed by many other viral species ${ }^{1,13,14}$.

The three canonical capping reactions responsible for the synthesis of the cap structure are outlined in 
a Chemical structure of the RNA cap

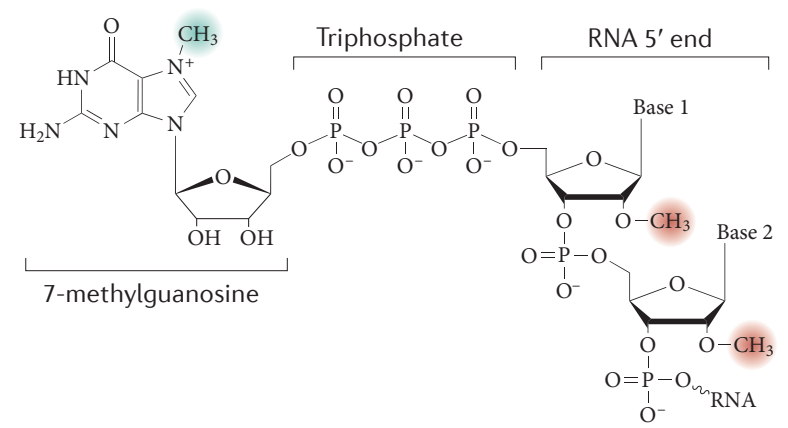

b Conventional RNA-capping
pathway

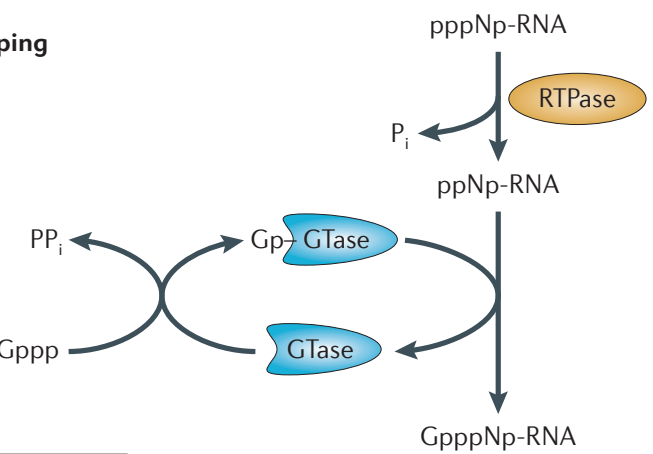

Significant viruses

- HIV (Retroviridae)

- HBV (Hepadnaviridae)

- HSV (Herpesviridae)

- Papillomaviruses (Papillomaviridae)

- Smallpox virus (Poxviridae)

- Rotaviruses (Reoviridae)

- BTV (Reoviridae)

- Yellow fever virus? (Flaviviridae)

- Dengue virus? (Flaviviridae)

- West Nile virus? (Flaviviridae)

- SARS CoV? (Coronaviridae)
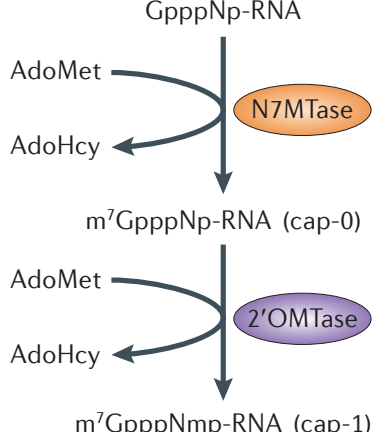

$m^{7} G p p p N m p-R N A$ (cap-1)

$\gamma$-phosphate

The third phosphate attached at the $5^{\prime}$ end of the ribose moiety of a nucleotide.

'Ping-pong' mechanism A two-step mechanism in which a substrate molecule first forms a (covalent) link with the enzyme and is then transferred to an acceptor molecule to yield a product.

Poly(A) tail

A string of AMP that is added to the $3^{\prime}$ end of mRNA.

NUDIX hydrolase superfamily A family of proteins that hydrolyse a wide range of organic pyrophosphates, including NDPs, NTPs, dinucleoside and diphosphoinositol polyphosphates, nucleotide sugars and RNA caps, with varying degrees of substrate specificity.
FIG. 1b. This pathway is found in all eukaryotic species, as well as in most DNA viruses and members of the family Reoviridae, and consists of the following reactions: hydrolysis of the $\gamma$-phosphate of the primary transcript by an RNA $5^{\prime}$-triphosphatase (RTPase); transfer of GMP to the $5^{\prime}$-diphosphate RNA (ppNp-RNA; in which $\mathrm{N}$ is the first transcribed nucleotide and $\mathrm{p}$ is a phosphate group) by a guanylyltransferase (GTase) through a 'ping-pong' mechanism, leading to the formation of GpppNp-RNA; and methylation of the guanosine moiety by a capspecific $S$-adenosyl-L-methionine (AdoMet)-dependent (guanine-N7)-methyltransferase (N7MTase), providing the minimal RNA cap chemical structure, named cap $-0\left(\mathrm{~m}^{7} \mathrm{GpppNp}\right)$, which is recognized by the translation factor eIF4 $\mathrm{E}^{15}$. Further methylation reactions catalysed by (nucleoside-2'-O)-methyltransferases ( $2^{\prime}$ OMTases) can occur on the first and second nucleotides $3^{\prime}$ to the triphosphate bridge, yielding cap-1 $\left(\mathrm{m}^{7} \mathrm{GpppNm} \mathrm{2}_{2^{\prime}-0}\right)$, and cap-2 $\left(\mathrm{m}^{7} \mathrm{GpppNm} \mathrm{2}_{2^{\prime}-0} \mathrm{pNm} \mathrm{2}_{2^{\prime}-0} \mathrm{p}\right)$ structures, respectively (FIG. 1). Cap-4 structures $\left(\mathrm{m}^{7} \mathrm{GpppNm}_{2^{\prime}-0} \mathrm{pNm}_{2^{\prime}-0} \mathrm{pNm}_{2^{\prime}-\mathrm{O}} \mathrm{pNm} \mathrm{2}_{2^{\prime}-\mathrm{O}} \mathrm{p}\right)$ were also identified on parasite mRNAs ${ }^{16}$.
Figure 1 | RNA cap structure and canonical capping mechanisms. a|The mRNA cap consists of a 7-methylguanosine linked to the 5' nucleoside of the mRNA chain through a $5^{\prime}-5^{\prime}$ triphosphate bridge. The methyl group at the N7 position of the guanosine is shaded green, and the $2^{\prime}$-O-methyl groups of the first and second nucleotide residues, forming the cap- 1 and the cap-2 structures, respectively, are shaded red. $\mathbf{b}$ |The cap- 0 structure is formed on nascent RNA chains by the sequential action of three enzymes. First, the RNA triphosphatase (RTPase) hydrolyses the $\gamma$-phosphate of the nascent RNA (pppNp-RNA, in which $\mathrm{N}$ denotes the first transcribed nucleotide and $p$ denotes a phosphate group) to yield a diphosphate RNA (ppNp-RNA) and inorganic phosphate $\left(P_{i}\right)$. Then, guanylyltransferase (GTase) reacts with the $\alpha$-phosphate of GTP (Gppp), releasing pyrophosphate $\left(\mathrm{PP}_{\mathrm{j}}\right)$ and forming a covalent enzyme-guanylate intermediate (Gp-GTase). The GTase then transfers the GMP molecule (Gp) to the $5^{\prime}$-diphosphate RNA to create GpppNp-RNA. In the final step, (guanine-N7)methyltransferase (N7MTase) transfers the methyl group from S-adenosyl-L-methionine (AdoMet) to the cap guanine to form the cap-0 structure, 7-methyl-GpppNp $\left(\mathrm{m}^{7} \mathrm{GpppNp}\right)$, and releases $\mathrm{S}$-adenosyl-L-homocysteine (AdoHcy) as a by-product. The capping reaction is completed by methylation of the ribose- $2^{\prime}-O$ position of the first nucleotide by the AdoMet-dependent (nucleoside-2'-O)-methyltransferase (2'OMTase), generating the cap-1 structure $\left(\mathrm{m}^{7} \mathrm{GpppNm} \mathrm{2}^{\prime-0} \mathrm{p}\right)$. The box contains examples of viruses that acquire their cap structures using the cellular capping machinery or encode their own viral capping machineries that adopt the canonical pathway. Question marks indicate viruses that are likely to follow this conventional pathway. The RNAs capped by viral enzymes are indistinguishable from cellular mRNA and can thus be translated into proteins by the cellular ribosomal machinery. BTV, bluetongue virus; $H B V$, hepatitis $B$ virus; $H S V$, herpes simplex viruses; SARS CoV, severe acute respiratory syndrome coronavirus.

Following translation, the lifespan of an mRNA molecule is controlled by two main processes in eukaryotic cells: first, the removal of its poly(A) tail and subsequent $3^{\prime}$-to-5' exonucleolytic degradation, and second, an mRNA-decapping step that allows 5'-to-3' exonucleolytic degradation (see REFS 17,18 for a review). Interestingly, decapped RNA may apparently be re-capped by the combined action of an as-yetunknown $5^{\prime}$-monophosphate kinase ${ }^{19}$ interacting with a host cell GTase that is also present in minor amounts in the cytoplasm (see REF. 20 for a review). Cytoplasmic decapping is catalysed by DCPS, a member of the NUDIX hydrolase superfamily, and stimulated by decapping enhancer proteins. Following RNA decapping in P-bodies, transcripts are quickly degraded by $5^{\prime}$-to- $3^{\prime}$ exonucleases such as XRN1. Thus, $5^{\prime}$-triphosphate mRNAs are almost completely absent in the cytoplasm. The eukaryotic cell has consequently evolved mechanisms to sense triphosphate RNA as non-self and uses these RNA species to trigger an innate immune response $e^{6,7}$. Viruses are the most common cell invaders to produce cytoplasmic mRNAs and have therefore been under a selective pressure to evolve 


\section{Box 1 | Getting around the lack of capping}

Some viruses (such as single-stranded positive-sense RNA (ss(+)RNA) viruses from the families Picornaviridae, Caliciviridae and Astroviridae) do not have a cap structure at the $5^{\prime}$ end of their mRNAs or genomic RNAs. Rather, they covalently attach to the 5' RNA end a protein termed $\mathrm{VPg}^{21}$ and/or carry an internal ribosome entry site (IRES) structure in the $5^{\prime}$ untranslated region ${ }^{154}$. IRESs have now been found in many different cellular and viral RNAs, including those of ss(+)RNA viruses from the families Picornaviridae and Dicistroviridae, the genus Lentivirus and the Flaviviridae genera Hepacivirus and Pestivirus. In pestiviruses, genomic RNA remains in a $5^{\prime}$-triphosphate form and thus promotes high levels of expression of host interferon-stimulated genes. However, these viruses also trigger several pathways that limit the antiviral response ${ }^{155,156}$, resulting in a competitive advantage for IRES-dependent translation of viral genes ${ }^{157}$. initiates translation of viral mRNAs ${ }^{21}$. In members of the Picornaviridae, VPg may be lost before translation ${ }^{22,23}$.

However, by far the most common viral mechanism for ensuring efficient translation of viral proteins and avoiding immune surveillance mechanisms is through the acquisition of a cap structure. The remarkable diversity of mechanisms that lead to an RNA cap structure identical to that of the host cell mRNAs is described below.

\section{Conventional capping of viral RNA}

Even when the viral replication cycle includes a nuclear phase, any virus entering a host cell must express its genes in the cytoplasm using the host translation machinery. Viral genomes can be made of singlestranded or double-stranded nucleic acids, either RNA or DNA, and the corresponding strategies used for protecting their RNA transcripts are outlined in FIG. 2.

Cap structures can be added to viral RNAs by one of the three following mechanisms. In the first mechanism, most viruses that synthesize their mRNA using cellular RNA pol II use the cellular capping machinery (FIGs 1b,2), as exemplified by most DNA viruses (except for those from the dsDNA virus family Poxviridae) and by RNA viruses such as those of the family Retroviridae (ss(+)RNA viruses) and the family Bornaviridae
Single-stranded positive-sense RNA viruses (ss(+)RNA viruses). Viruses that have or produce mRNAs that are co-linear to their genomic RNA.

43S pre-initiation complex A multiprotein complex composed of eukaryotic translation initiation factor 3 (elF3), elF4A, elF4E and elF4C associated with the small ribosomal subunit. This pre-initiation complex scans the mRNA towards the 'start' codon (typically AUG), where translation is initiated. counteracting mechanisms to conceal their RNA $5^{\prime}$ ends from the innate immunity machineries of the host cell.

Some viruses, such as those in the family Picorna -stranded positive-sense RNA viruses (ss $(+)$ complex in a $5^{\prime}$-cap-independent manner (BOX 1). Other viruses (for example, viruses of the family Caliciviridae, which are also ss(+)RNA viruses) covalently attach their RNA 5' end to a VPg-like protein, which directly interacts with the cap-binding protein eIF4E and

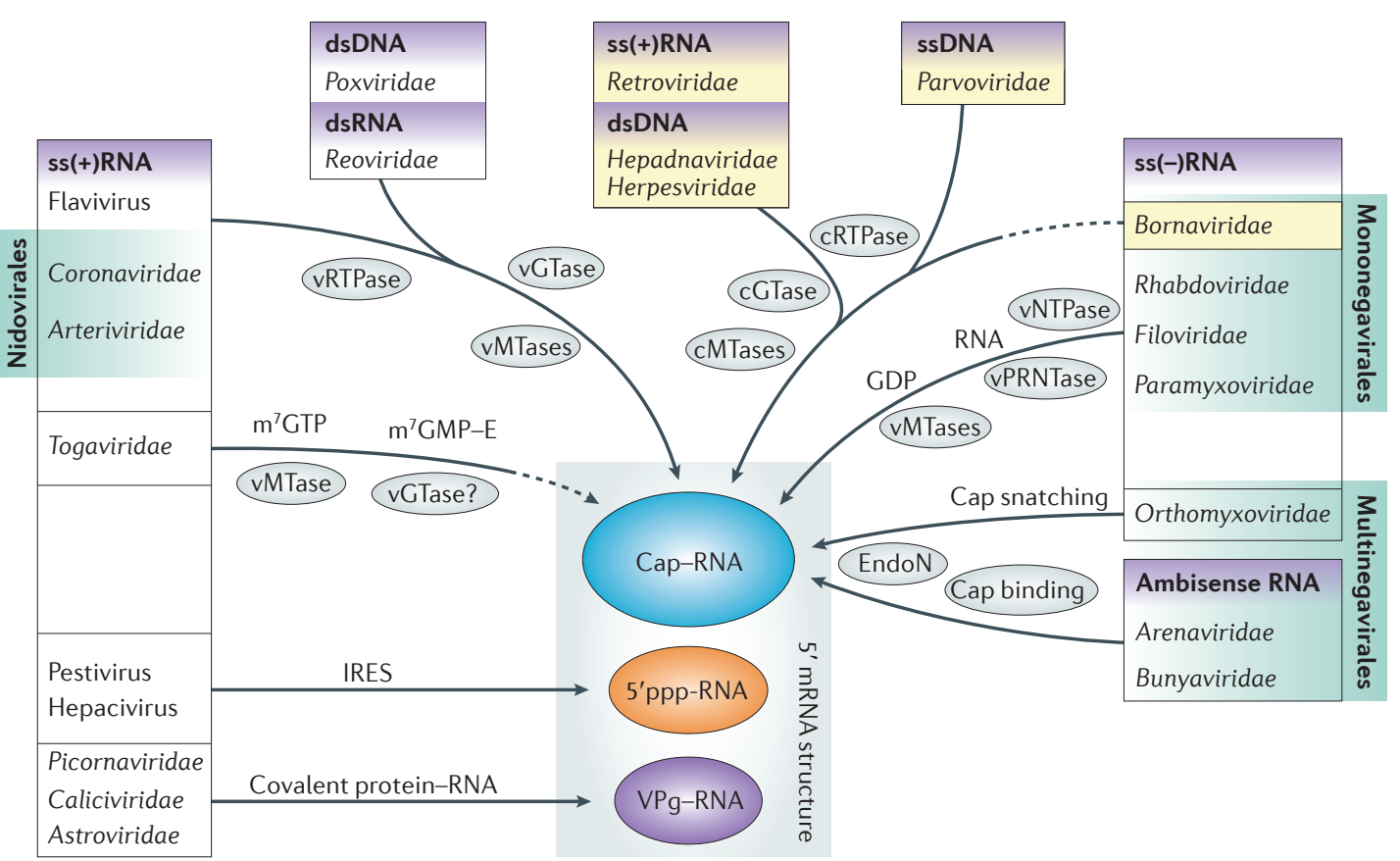

Figure 2 | RNA 5' ends in the mammalian-virus world. Mammalian viruses, with the exception of those from the single-stranded positive-sense RNA (ss(+)RNA) virus genera Pestivirus and Hepacivirus, use strategies to chemically modify their mRNA 5' ends through either covalent attachment of a protein (VPg for ss(+)RNA viruses from the families Picornaviridae, Caliciviridae and Astroviridae) or covalent attachment of an RNA cap structure (all other viruses). Arrows indicate the type of RNA 5' end protection that is used by these viral groups, and the enzymes and mechanisms involved are indicated. Dashed arrows indicate a likely but incompletely demonstrated pathway. Viral and cellular proteins are distinguished with the prefixes $v$ and c, respectively. Yellow shading highlights viral groups for which the life cycle includes a nuclear phase in the host cell. This list of viral taxa is non exhaustive and used as an example only. ds, double-stranded; E, enzyme; EndoN, endonuclease; GTase, guanylyltransferase; IRES, internal ribosome entry site; m , 7-methyl; MTase, methyltransferase; NTPase, nucleotide 5'-triphosphatase; $p$, phosphate group; PRNTase, polyribonucleotidyl transferase; RTPase, RNA triphosphatase; ss(-)RNA, single-stranded negative-sense RNA. 
a Rhabdoviridae-like

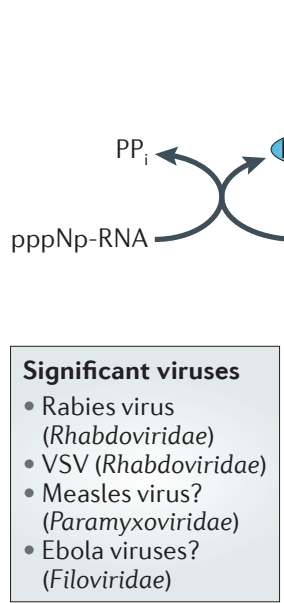

Gppp

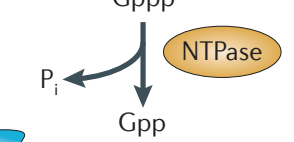
RNTase-pNp-RNA

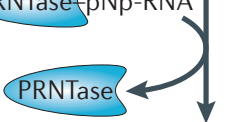

GpppNp-RNA

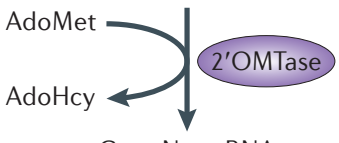

GpppNmp-RNA

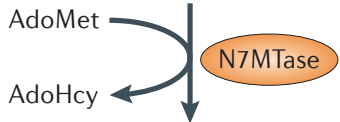

b Alphaviridae-like

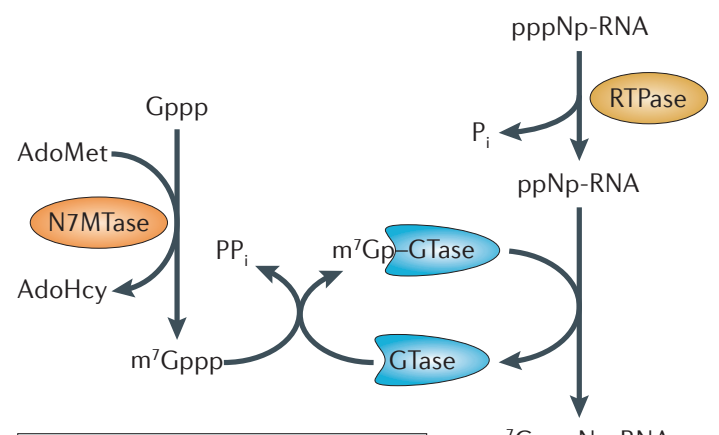

$\mathrm{m}^{7}$ GpppNp-RNA

$\mathrm{m}^{7} \mathrm{GpppNmp-RNA}$

Significant viruses

- Chikungunya virus? (Togariridae)

- Rubella virus? (Togaviridae)

- HEV? (Hepeviridae)

\section{c Orthomyxoviridae-like}
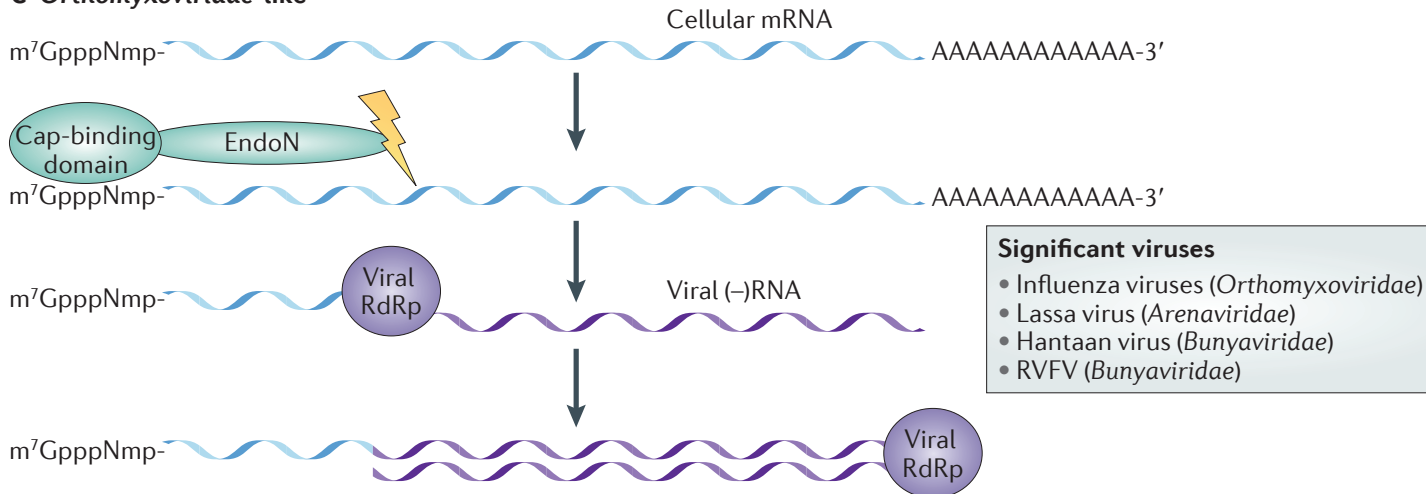

Figure 3 | Unconventional capping pathways. a | The RNA-capping mechanism of negative-sense RNA ((-)RNA) viruses such as those of the family Rhabdoviridae. The NTPase hydrolyses the $\gamma$-phosphate of GTP (Gppp) to yield a GDP (Gpp) and inorganic phosphate $\left(P_{i}\right)$. Polyribonucleotidyl transferase (PRNTase) reacts with the nascent viral RNA (pppNp-RNA; in which $\mathrm{N}$ denotes the first transcribed nucleotide), releasing pyrophosphate $\left(\mathrm{PP}_{\mathrm{i}}\right)$ and forming a covalent PRNTase-pNp-RNA intermediate. The PRNTase then transfers the RNA molecule to the GDP to create GpppNp-RNA. (Nucleoside-2'-O)methyltransferase (2'OMTase) transfers the methyl group from S-adenosyl-L-methionine (AdoMet) to the first nucleotide of the RNA. The capping reaction is then completed by methylation of the cap by the AdoMet-dependent (guanine-N7)methyltransferase (N7MTase). The box lists examples of viruses that acquire their cap structures using such a capping pathway. Question marks indicate viruses that are likely to follow this conventional pathway. b | The RNA-capping mechanism of positive-sense RNA ((+)RNA) viruses such as those of the family Alphaviridae. The RNA triphosphatase (RTPase) hydrolyses the $\gamma$-phosphate of the viral RNA to yield a diphosphate RNA (ppNp-RNA) and P. A GTP molecule is methylated at its N7 position by the AdoMet-dependent N7MTase. Guanylyltransferase (GTase) then binds the N7-methyl-GTP $\left(\mathrm{m}^{7} \mathrm{Gppp}\right)$, forming a covalent link with a catalytic histidine $\left(\mathrm{m}^{7} \mathrm{Gp}-\mathrm{GTase}\right)$ and releasing PP. The GTase then transfers the $m^{7} \mathrm{GMP}$ molecule ( $\mathrm{m}^{7} \mathrm{Gp}$ ) to the $5^{\prime}$-diphosphate RNA to create $\mathrm{m}^{7} \mathrm{GpppNp}-\mathrm{RNA}$. The box indicates viruses that acquire their cap structures using such a capping pathway. $\mathbf{c}$ | the RNA-capping mechanism of (-)RNA viruses such as those of the family

Single-stranded negative-sense RNA viruses (ss(-)RNA viruses). Viruses that have or produce mRNAs that are complementary to their genomic RNA.

Ambisense RNA viruses Viruses (such as members of the families Arenaviridae and Bunyaviridae) that have or produce both mRNAs that are co-linear to and mRNAs that are complementary to their genomic RNA, although most mRNAs are complementary in polarity.

Orthomyxoviridae; this mechanism is referred to as cap snatching. The PB2 subunit of the viral RNA-dependent RNA polymerase (RdRp) binds to the $5^{\prime}$ end of cellular capped mRNAs (which are enriched in the processing (P)-bodies), and the PA subunit then releases short capped RNAs by using its endonuclease (EndoN) activity. These capped RNAs are used as primers by the viral RdRp in viral transcription to generate viral mRNA using the viral (-)RNA as a template. The RdRp then synthesizes the complementary negative-sense strand. The box provides example of viruses that acquire their cap structures using a similar capping pathway. Note that most of the mRNAs that are capped by viral enzymes are indistinguishable from cellular mRNAs and can be translated into proteins by the cellular ribosomal machinery. AdoHcy, S-adenosyl-L-homocysteine; HEV, hepatitis E virus; RVFV, Rift Valley fever virus; VSV, vesicular stomatitis virus.

(single-stranded negative-sense RNA viruses (ss(-)RNA viruses)). A second strategy consists of acquiring cap structures from cellular mRNAs by 'cap snatching' (FIGS 2,3). RNA viruses such as members of the families Orthomyxoviridae, Arenaviridae and Bunyaviridae
— which are ss(-)RNA viruses, with the latter two families also referred to as ambisense RNA viruses - steal short, capped cellular mRNAs through endonucleolytic cleavage. The stolen short, capped mRNAs are then used by the viral polymerase to prime synthesis of viral RNA. 
For the third method, many viruses encode their own capping machinery and have evolved a diverse set of dedicated enzymes and mechanisms to carry out capping. Accordingly, most viruses with an ssRNA genome synthesize or acquire the RNA cap using their own set of enzymes (FIG. 2). Within this diversity, the capping of viral mRNA can be classified as either 'conventional', when it follows the mRNA-capping pathways used by eukaryotes and DNA viruses (that is, the sequential action of RTPase, GTase and MTases) (FIGS 1b,2), or 'unconventional', when it does not (see below and FIG. 3).

The best characterized conventional RNA-capping system is that exemplified by the dsDNA virus vaccinia virus, which expresses a multifunctional mRNA capsynthesizing enzyme (D1) containing RTPase, GTase and N7MTase domains ${ }^{24}$. The $5^{\prime}$-triphosphate of the nascent mRNA is first hydrolysed by the RTPase to yield 5 -diphosphate RNA, which is then sequentially transferred to other internal domains ${ }^{25,26}$ to be capped and methylated, the latter reaction with allosteric stimulation through direct association with viral D12 $\operatorname{protein}^{25,26}$. Cap assembly is completed by the viral VP39, a bifunctional protein that catalyses the 2 '-O-methylation of the cap-0 structure and also acts as an elongation factor for poly(A) polymerase ${ }^{27}$. Viruses from the dsRNA virus family Reoviridae share the same pathway as vaccinia virus. The enzymes remain physically associated in an inner capsid (or 'transcriptionally active core'), which constitutes a molecular machine or 'assembly line' that is able to transcribe the genome, synthesize the cap and inject the resulting mRNA into the cytoplasm of the host cell for translation. In addition, further assignment and characterization of the GTase activity in viruses of the genus Flavivirus and those of the family Coronaviridae may join these viruses, which are ss(+)RNA viruses, to the conventional RNA-capping pathway group.

The second phosphate attached at the $5^{\prime}$ end of the ribose moiety of a nucleotide.

Walker A and B motifs Motifs that are present in nucleotide-binding proteins but also in a range of proteins with widely varying functions, including ATP synthases, myosins, transducins, helicases, kinases and RecA proteins. The Walker A motif contains a phosphate-binding loop (P-loop) motif with the consensus sequence GXXXGK(T/S), and the Walker B motif contains the consensus sequence (R/K)XXXXGXXXXLhhhhD (in which $h$ refers to any hydrophobic residue).

Nucleophilic attack Generally, a starting point for a chemical reaction; a doublet of electrons selectively attacks the positive or partially positive charge of the atomic nucleus in order to create a new chemical bond. between the two domains, which contains a histidine residue involved in binding and in $\mathrm{Mg}^{2+}$-dependent hydrolysis of both NTP and RNA substrates. NSP2 selfassembles into a doughnut-shaped octamer, a quaternary structural organization that creates several RNA-binding sites, which are presumably needed to destabilize RNA duplexes during genome replication and packaging ${ }^{30,32,33}$.

TTM enzymes hydrolyse NTPs in the presence of $\mathrm{Mn}^{2+}$ or $\mathrm{Co}^{2+}$ and are found in fungi and protozoa, and in most DNA viruses that encode an RTPase, including poxviruses, chlorella virus, baculoviruses and mimiviruses $^{34-37}$. The RTPase Cet1 from Saccharomyces cerevisiae is nearly identical to that of mimiviruses and serves as a paradigm for the TTM group. Its structure features a characteristic tunnel lined by eight antiparallel $\beta$ strands (FIG. 4a) and a double glutamate motif ${ }^{36,38}$. The mRNA supposedly sits in the tunnel, which harbours several charged and hydrophilic side chains that coordinate $\mathrm{Mn}^{2+}$ and $\mathrm{SO}^{2-}$ in the crystal structure. $\mathrm{SO}^{2-}$ is thought to indicate the position of the $\gamma$-phosphate of mRNA.

The third group comprises proteins with NTPase and helicase activity, belonging to the large helicase superfamilies SF1 and SF2 (REFS 39-41). Such NTPase-helicase family members are found in flaviviruses (for example, NS3 proteins of dengue virus, yellow fever virus, West Nile virus and Japanese encephalitis virus), coronaviruses (for example, nsp13 of severe acute respiratory syndrome coronavirus (SARS CoV), although for this member of the SF1 helicases the crystal structure is not known), alphaviruses (for example, the protein nsP2) and potexviruses (for example, protein $1 \mathrm{~A}$ of bamboo mosaic virus), all of which are ss(+)RNA viruses, and also in viruses of the family Reoviridae (for example, the protein $\lambda 1$ ), which are dsRNA viruses. The fold adopted by SF2 helicases features two RecA-like subdomains between which a cleft accommodates the nucleotide or 5 '-triphosphate RNA substrate ${ }^{42}$. The RecA subdomains I and II carry the Walker A and B motifs. Residues belonging to the Walker A motif (also named motif I) form the P-loop, which stabilizes the terminal phosphate moiety of the substrate, whereas acidic residues from the Walker B motif (also named motif II or DEXD box) coordinate the $\mathrm{Mg}^{2+}$ needed for hydrolysis ${ }^{42}$. Structural and biochemical studies revealed that the RTPase and NTPase activities of helicase enzymes from flaviviruses (ss(+)RNA) have a common catalytic site $28,42-47$. Similar biochemical studies have mapped an associated helicase-RTPase activity in other viral families, including alphaviruses $(\mathrm{ss}(+) \mathrm{RNA})^{48}$. However, structural data are needed to better understand how these multifunctional viral proteins coordinate their various activities.

Metal-independent RTPases are found in plants, metazoa and viruses such as baculoviruses (dsDNA) ${ }^{49,50}$. They belong to the cysteine phosphatases superfamily. The RTPase reaction proceeds in two steps, starting with entry of the $5^{\prime}$-triphosphate RNA into the activesite tunnel and formation of a covalent cysteinyl$S$-phosphoester adduct. The catalytic cysteine responsible for the nucleophilic attack on the $\gamma$-phosphate of the RNA $5^{\prime}$ end belongs to a P-loop motif (HCXXXXXR(T/S)). The second step releases inorganic phosphate. Cysteine 


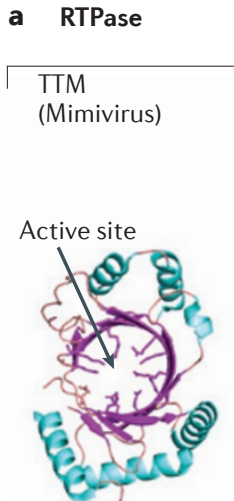

Metal-independent mechanism

(Baculoviruses)

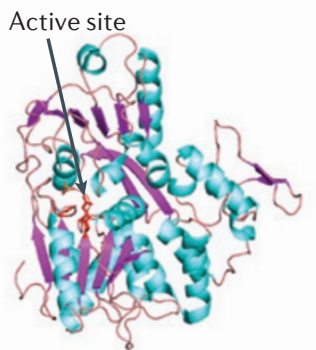

Helicase

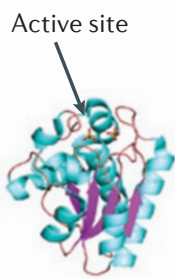

b GTase
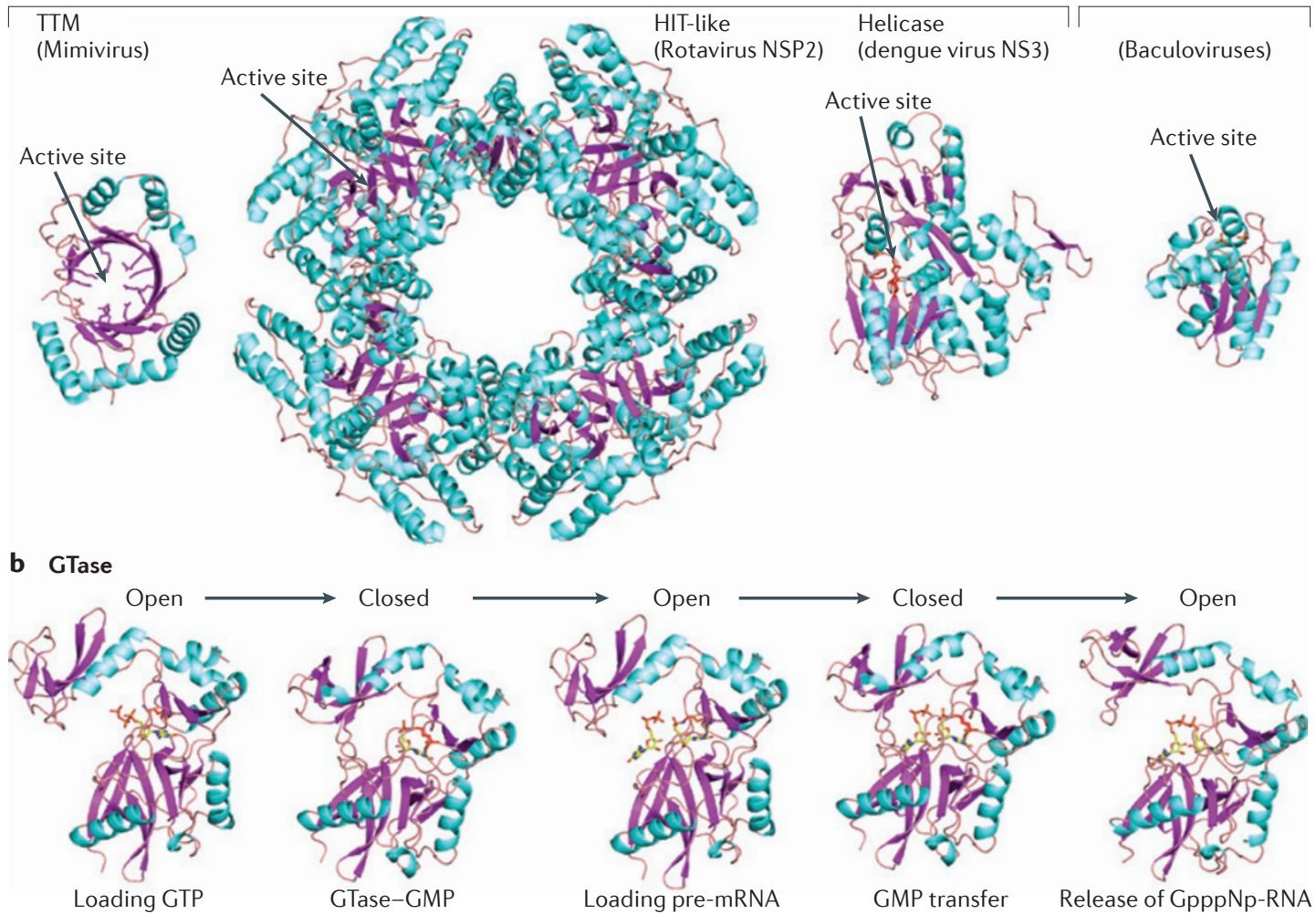

c Assembly line

Cossembly line

RTPase and GTase

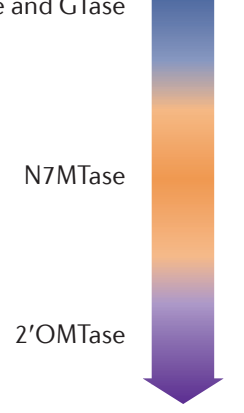

Reaction sequence

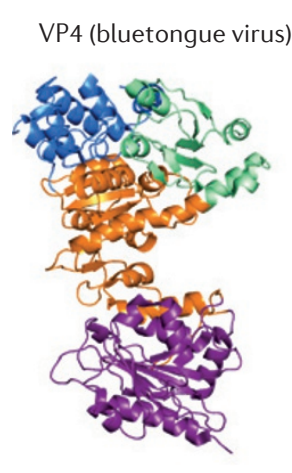

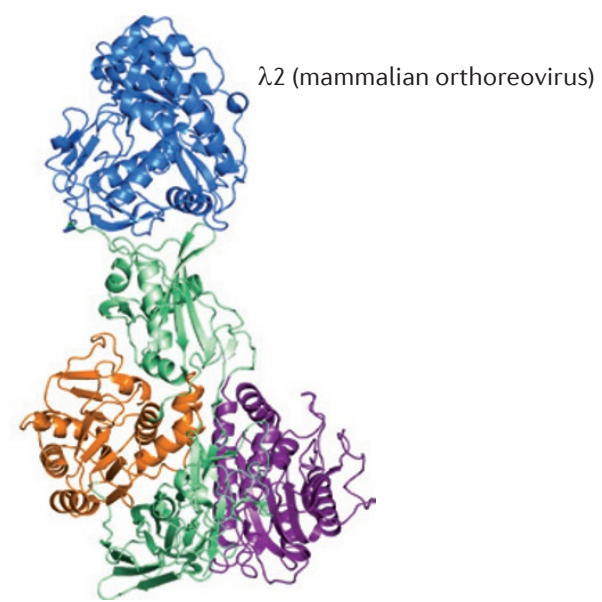
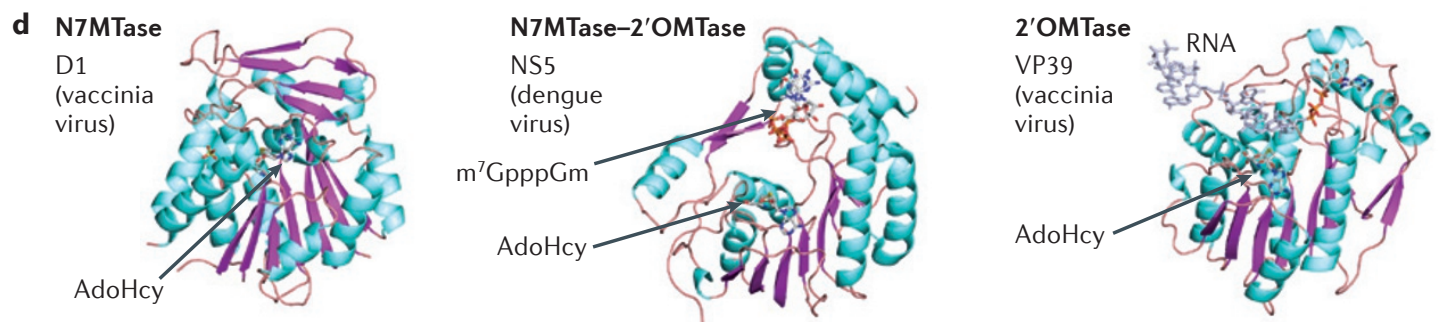

a-phosphate

The first phosphate attached at the $5^{\prime}$ end of the ribose moiety of a nucleotide. phosphatases adopt a characteristic $\alpha-\beta$ fold with a central twisted, five-stranded parallel $\beta$-sheet flanked by six $\alpha$-helices ${ }^{49}$. The catalytic cysteine that specifically recognizes the $\gamma$-phosphate of the RNA $5^{\prime}$ end resides at the bottom of the substrate-binding pocket, whereas other conserved residues from the P-loop, together with surrounding residues, form a positively charged channel that can accommodate the $\alpha$-phosphate and the $\beta$-phosphate of the RNA $5^{\prime}$ end. The shape of the binding pocket dictates the selectivity for triphosphate RNA and seems too deep to grant diphosphate RNA access to the active site. 
4 Figure 4 | Structural constituents of viral capping machineries, folds and mechanisms. a | Different RNA triphosphatase (RTPase) folds. For the metal-dependent mechanism, the triphosphate tunnel metalloenzyme (TTM) fold is exemplified by the structure of the RTPase (Protein Data Bank (PDB) accession code 2QZE) from the genus Mimivirus, which consists of double-stranded DNA (dsDNA) viruses; the histidine triad (HIT)-like fold of the RTPase from dsRNA rotaviruses (PDB accession code $1 \mathrm{~L} 9 \mathrm{~V}$ ) and the helicase fold of the RTPase from the single-stranded positive-sense RNA (ss(+)RNA) virus dengue virus (PDB accession code 2BHR) are also shown. For the metal-independent mechanism, the fold of the RTPase from the dsDNA baculoviruses (PDB accession code 1 YN9) is the only viral structure available so far. Structures are coloured cyan for $\mathbf{a}$-helices and pink for $\beta$-strands. $\mathbf{b}$ | Structure-based model of a guanylyl transfer mediated by the dsDNA virus Paramecium bursaria chlorella virus 1 guanylyltransferase (GTase). Presented are the five stages of the reaction: loading of GTP onto the active site, with the GTase in an open conformation (PDB accession code 1CKO); the GTase in a closed conformation and the creation of covalent intermediate GTase-GMP through a lysine residue (model derived from the structure in PDB accession code $1 \mathrm{CKN}$ ); the intermediate GTase-GMP reopening to bind a pre-mRNA molecule (model derived from the structure in PDB accession code 1CKO); the GTase closing again to complete the transfer (model derived from the structure in PDB accession code 1CKN); and, transfer completed, the GTase opening to release the GpppNp-RNA (in which p is a phosphate group and $\mathrm{N}$ is the first transcribed nucleotide) (PDB accession code $1 \mathrm{CKM}$ ). Structures are coloured cyan for $\alpha$-helices and pink for $\beta$-strands. c $\mid$ Assembly line structures. Protein VP4 (PDB accession code 2J J C) of bluetongue virus (a dsRNA virus from the genus Orbivirus) and protein $\lambda 2$ of mammalian orthoreovirus (a dsRNA virus) (PDB accession code 1EJ6). The colour code correlates with the colour of each domain as represented in the assembly sequence arrow to the left. Domains in green are extra domains that do not take part directly in the capping mechanism. d | Methyltransferase structures. Left: the (guanine-N7)-methyltransferase (N7MTase) domain of protein D1 from the dsDNA virus vaccinia virus (PDB accession code 2VDW) in complex with a molecule of S-adenosyl-L-homocysteine (AdoHcy). Middle: the N7MTase-(nucleoside2'-O)-methyltransferase (2OMTase) domain of NS5 from dengue virus (ss(+)RNA) in complex with the cap analogue 7-methyl-G $\left(\mathrm{m}^{7} \mathrm{G}\right)-\mathrm{pppGm} \mathrm{2}_{2^{\prime}-\mathrm{O}}$ and AdoHcy (PDB accession code 2P41). Right: VP39, the 2'OMTase of the dsDNA virus vaccinia virus, in complex with a capped RNA and AdoHcy (PDB accession code $\underline{1 \mathrm{AV} 6}$ ). All figures were prepared using PyMOL.

$\mathrm{p} K_{\mathrm{a}}$ value

The acid dissociation constant,

a quantitative measurement of the strength of a chemical group as an acid in solution. It corresponds to the $\mathrm{pH}$ value at which half of the ionizable group is either protonated or deprotonated.

$\varepsilon$-amino group A positively charged group found at the extremity of a lysine side chain. The $\varepsilon$-amino group is a primary amine and, owing to its high $p K_{a}$ value, it is reactive and often participates in reactions at the active site of enzymes.
Guanylyltransferases. GTases of DNA viruses contain two domains: a nucleotidyltransferase (NTase) domain that is conserved in capping enzymes, RNA ligases and DNA ligases ${ }^{51}$, and a C-terminal oligonucleotide-binding domain that is observed in capping enzymes and several DNA ligases. Sequence alignments aided by structural information for several family members identified conserved residues and motifs both in the nucleotide-binding site and in the NTase site ${ }^{51,52}$. A lysine-containing motif, $\mathrm{KXDG}(\mathrm{I} / \mathrm{L})$, is conserved among the GTases encoded by several DNA viruses (vaccinia virus, Shope fibroma virus and African swine fever virus) and the yeasts $S$. cerevisiae and Schizosaccharomyces pombe. The lysine in this motif was shown to be the catalytic residue in the GTase of vaccinia virus ${ }^{53-55}$ and in the yeast capping enzyme ${ }^{56}$. Moreover, this motif is conserved in the active site of polynucleotide ligases, which, like capping enzymes, catalyse an enzymatic reaction via the formation of a covalent Lys-NMP intermediate ${ }^{57}$.

The structure of the GTases from Paramecium bursaria chlorella virus 1 (dsDNA), humans ${ }^{58}$ and the yeast Candida albicans (the protein Cgt1) have been solved individually and, for the chlorella virus GTase and Ctg1, in complexes with cap analogues, GTP or as a covalent GTase-GMP intermediate ${ }^{59-61}$. These structures suggest the following reaction scheme, during which the oligonucleotide-binding and NTase domains, acting as a clamp, undergo a series of large conformational changes. These domains open up to load GTP, close to catalyse the formation of a covalent enzyme-GMP complex through the action of the catalytic lysine, open again to release pyrophosphate and bind the pre-mRNA substrate, close again to catalyse nucleotidyl transfer to the RNA and finally re-open to release the GpppNp-RNA product ${ }^{51,60}$ (FIG. 4b).

The dsRNA viruses of the family Reoviridae use one multifunctional capsid protein for the capping reaction. $\lambda 2$ of the dsRNA virus mammalian orthoreovirus ${ }^{62}$, VP4 of bluetongue virus (in the genus Orbivirus ${ }^{63}$ ) and VP3 from cytoplasmic polyhedrosis virus (in the genus Cypovirus ${ }^{64}$ ) act as assembly lines, such that the RNA substrate is shuttled from one domain to the next (see below). The GTase domains of these proteins feature different folds (see below and FIG. 4c) to DNA virus GTases. Despite these structural differences, viruses from the genera Orthoreovirus and Aquareovirus use a conserved lysine residue (although not part of the signature sequence found in DNA viruses) to form a covalent intermediate with GMP ${ }^{65,66}$. In flaviviruses (ss(+)RNA), the 2'OMTase domain has been proposed to act as a GTase $^{67,68}$. However, the proposed catalytic lysine is not conserved in flaviviruses ${ }^{69}$.

Methyltransferases. Despite the limited overall amino acid sequence identity in the large family of AdoMetdependent MTases, most of these enzymes share a common structural core made of seven $\beta$-strands flanked by three $\alpha$-helices on each side of the sheet, similar to the core found in the catechol-O-MTase (a class I AdoMet-dependent MTase $\left.\mathrm{e}^{70,71}\right)$. This core catalytic domain has evolved extensions that consist of structurally non-conserved domains and allow these MTases to accommodate a range of methyl acceptors $^{70}$. The MTases exist either as isolated proteins (for example, nsp16 viruses from the Coronaviridae; $\mathrm{ss}(+) \mathrm{RNA}^{72}$ ), or as domains of larger proteins (such as NS5 of viruses from the ss(+)RNA virus genus Flavivirus ${ }^{69,73}$ or the multidomain cap assembly lines of dsRNA viruses from the Reoviridae ${ }^{62}$ ). In some instances the same protein domain can have dual N7MTase and 2'OMTase activities (for example, the MTase domain in NS5 from flaviviruses ${ }^{74,75}$ ), sharing the same cofactor-binding site. When this is the case, repositioning of the RNA substrate must occur.

Both N7MTases and 2'OMTases share the class I family fold (FIG. 4d). N7-methyl transfer is thought to be promoted by optimal positioning of the reacting groups, mediated by several aromatic residues, and by an electrostatic environment that is favourable for the reaction ${ }^{25,76}$. By contrast, 2'OMTases rely on a conserved catalytic tetrad, Lys-Asp-Lys-Glu ${ }^{69,77,78}$. The catalytic reaction was deciphered by several structural studies of the 2'OMTase VP39 of vaccinia virus ${ }^{79,80}$. It was suggested that residues in the vicinity of the catalytic Lys175 (the second lysine in the Lys-Asp-Lys-Glu motif) decrease the $p K_{a}$ value of its $\varepsilon$-amino group. This orientates the 2 -hydroxyl group of the ribose for a nucleophilic in-line $s n-2$ attack on the AdoMet methyl group ${ }^{81}$. 
Using cap analogues, the molecular basis for recognition of GpppNp-RNA versus cap-0-RNA has also been characterized for VP39 (REFS 80,82). The $\mathrm{m}^{7} \mathrm{G}$ is stacked between two aromatic residues, and electron delocalization and electrostatically enhanced stacking owing to N7 methylation favours the recognition of the cap over GTP (FIC. 4d).

One interesting aspect of MTase activity relates to its regulation: in three cases, interfacial activation is achieved either by a cofactor protein (such as the vaccinia virus D12 subunit, which enhances the N7MTase activity of D1 through an allosteric mechanism ${ }^{25,26}$, and the SARS CoV (ss(+)RNA) metalloprotein nsp10, which acts as a cofactor to activate the 2'OMTase nsp16 but not the N7MTase nsp14 (REF. 83)) or by binding to lipid membranes (as is the case for nsP1 of the ss(+)RNA alphaviruses).

What determines the sequence of the two methylation steps? For flaviviruses ${ }^{74,75}$ or coronaviruses ${ }^{83,84}$, which are ss(+)RNA viruses, the order in which methylations are performed is not encoded in the global protein architecture. Rather, variations in kinetics and affinity may dictate the order in which reactions occur ${ }^{83,85}$. In the case of flaviviruses, RNA secondary structures also seem to be important: whereas the N7MTase activity of the bifunctional NS5 MTase domain requires a long substrate encompassing a specific stem loop RNA structure, christened stem loop A (SLA), the 2'OMTase activity is able to transfer a methyl group to short RNA acceptors ${ }^{75}$.

Cap assembly lines. Several dsRNA viruses encode structural proteins that are packaged with their genome in the viral particle and are able to perform the four reactions needed to synthesize a cap-1 structure, much like an assembly line (FIG. 4c). The key molecular components of the RNA-capping machinery in members of the dsRNA virus family Reoviridae are RNA-directed RNA polymerase (named VP1 in orbiviruses and rotaviruses, and $\lambda 3$ in orthoreoviruses) and a multifunctional cap-synthesizing enzyme (named VP4 in orbiviruses, VP3 in rotaviruses and $\lambda 2$ in orthoreoviruses). Both $\lambda 2$ and VP4 are composed of four domains that were identified as RTPase, GTase, N7MTase and 2'OMTase, respectively ${ }^{62,63}$. The spatial arrangement of the different protein domains reflects the time sequence of the enzymatic reactions that are required for mRNA capping following synthesis by the viral polymerase (FIG. 4C). Although it is unclear how and when RTPase activity occurs, a complete pathway has been proposed in which guanylyl transfer occurs near the base of the pentameric 'turret' (formed by $\lambda 2$ in orthoreoviruses), followed by N7-methylation and 2'-O-methylation of the mRNA ${ }^{62}$. Therefore, in dsRNA viruses, the sequence of steps in the cap synthesis pathway should remain identical to the sequence in the capping pathway for cellular mRNAs and for DNA viruses such as vaccinia virus, for which the capping machinery is embedded in a multidomain protein complex.

The GTase domain and both MTase domains of bluetongue virus (a dsRNA virus in the genus Orbivirus) were unambiguously mapped on the VP4 structure, but the position of the RTPase domain remains uncertain ${ }^{63}$. However, it is believed that both the RTPase and GTase activities reside in the same C-terminal domain of VP4, a unique architecture that is reminiscent of, but distinct from, double-domain RTPase-GTase proteins found in metazoans and plants. The enzymatic activity requires $\mathrm{Mg}^{2+}$ (REFS 63,86,87). However, VP4 does not adopt a typical metal-dependent RTPase fold. A cysteine residue is found in a deep cavity similar to that harbouring the catalytic motif of the cysteine phosphatase superfamily ${ }^{63}$. Thus, these assembly line enzymes seem to have incorporated features from various phylogenetic origins.

\section{Unconventional cap synthesis pathways}

The first indication that there are deviations from the conventional RNA-capping pathway for viral mRNAs came in the early 1970s, around the time of the discovery of the RNA cap structure. Since then, is has been demonstrated that the ss(-)RNA virus vesicular stomatitis virus (VSV) and ss(+)RNA alphaviruses (from the family Togaviridae) can synthesize a viral RNA cap that is identical to a cellular RNA cap, albeit constructed through a completely different mechanism. Although alphaviruses do not proceed further than synthesizing a cap-0 structure, the fact that divergent biosynthetic pathways converge to the consensus cap structure indicates that the selective pressure to maintain this structure must be high.

The Mononegavirales RNA-capping pathway. Mononegavirales is a viral order of ss(-)RNA viruses with unsegmented genomes, such as VSV and rabies virus (in the family Rhabdoviridae), measles virus (from the family Paramyxoviridae), bornavirus (from the family Bornaviridae), and Ebola viruses and Marburg viruses (from the family Filoviridae). These viruses encode a multifunctional L protein that carries RNA-dependent RNA polymerase (RdRp) and RNA cap synthesis activities. These enzymes have evolved independently from other known eukaryotic cap-synthesizing enzymes, and the L proteins of $\mathrm{VSV}^{88,89}$, spring viraemia of carp virus ${ }^{90}$, human respiratory syncytial virus ${ }^{91}$ and Chandipura virus ${ }^{92}$ transfer GDP rather than GMP to the RNA 5' end. Part of a domain in the conserved region $\mathrm{V}$ of $\mathrm{L}$ protein contains the GDP polyribonucleotidyl transferase (PRNTase) activity, and forms a covalent enzyme-pNp-RNA intermediate (FIG. 3a) with the nascent viral RNA. The covalent bond with RNA involves a conserved histidine residue present in an 'HR' motif instead of the lysine residue used by conventional GTases $^{92}$. The 5'-monophosphorylated viral mRNA start sequence then receives GDP generated from $\mathrm{GTP}^{93}$ by an as-yet-unknown NTPase. The VSV MTase, present in domain VI of L protein ${ }^{78,94}$, subsequently methylates the core cap structure at the ribose- $2^{\prime}-O$ position of the first nucleotide, followed by methylation at the guanine-N7 position, generating GpppAm $\mathrm{2}_{2^{\prime}-\mathrm{O}}$-RNA and $\mathrm{m}^{7} \mathrm{GpppAm}_{2^{\prime}-\mathrm{O}}-\mathrm{RNA}$, respectively ${ }^{95-97}$. The capping reaction seems to be dependent on RNA length, indicating a possible spatial rearrangement in L protein ${ }^{98}$. Although no crystal structure is available yet, the MTase activities 


\section{Box 2 | The RNA-decapping pathway of viruses}

Viruses cap and decap RNA, and many viruses regulate the decapping pathway in order to control the ratio of viral and cellular mRNAs.

Decapping of cellular mRNA by Saccharomyces cerevisiae L-A virus (from the Totiviridae family of double-stranded RNA viruses) proceeds through a decapping enzyme carried by the Gag subunit of the capsid; this Gag subunit is responsible for covalently binding cap structures (7-methyl-GpppG $\left(\mathrm{m}^{7} \mathrm{GpppG}\right)$, in which $\mathrm{p}$ is a phosphate group) of cellular mRNA ${ }^{158}$. The decapping activity of Gag aids in the expression of viral RNA, apparently by producing large amounts of cellular RNA decoys that inhibit the $S$. cerevisiae enzyme $5^{\prime}-3^{\prime}$ exoribonuclease $1\left(X_{\mathrm{rn}} 1\right)^{159}$ and compete with degradation of viral RNA. How the viral mRNA is recruited by the eukaryotic translation initiation factor $4 \mathrm{E}$ (elF4E) complex remains to be elucidated. In the case of the family Poxviridae (double-stranded DNA viruses), the decapping enzyme (D10) increases the turnover of host mRNAs and contributes to the shutdown of host protein expression ${ }^{160}$. Moreover, D10 seems to preferentially degrade $m^{7} \mathrm{GpppGm}_{2^{\prime}-\mathrm{O}}$ rather than $m^{7} G p p p A m_{2^{\prime}-0}$ and thereby hydrolyses early-phase viral RNA carrying predominantly $\mathrm{m}^{7} \mathrm{GpppGm} \mathrm{2}_{2^{\prime}-\mathrm{O}}$ cap structures ${ }^{161}$. In other words, this viral pathway benefits from having mRNAs (produced by the capping apparatus) that will be recruited by the elF4E complex, and simultaneously removes the potential competition from cellular mRNAs for ribosome binding. Viral 'cap-snatching' (see main text) also results in this imbalance, favouring expression of viral genes. Finally, several viruses have been reported to interfere with the cellular RNA-trafficking and decoy machinery. First, a single-stranded positive-sense RNA (ss(+)RNA) enterovirus was shown to inhibit the ability of cells to form stress granules by cleaving RAS.GAP-binding protein (G3BP) family members. The kinetics of poliovirus-induced processing (P)-body disruption correlates with production of viral proteases that induce the degradation of P-body proteins, such as DCP1A and the 3'-deadenylase complex component PAN3. Recently, the ss(+)RNA viruses hepatitis $C$ virus and HIV were also reported to be connected to the cellular decapping machinery and to regulate it (reviewed in REF. 162).

Cell-based replicon assays Assays that allow one to follow the replication of a 'minimal viral genome' encoding the viral replication complex but no structural or envelope proteins, which are usually replaced by reporter genes (such as luciferase or chloramphenicol acetyl transferase genes). share the AdoMet-binding and active site, which comprises a conserved Lys-Asp-Lys-Glu catalytic tetrad, typical of $2^{\prime} \mathrm{OMT}$ Tases.

The RNA-capping pathway of the alphavirus-like togaviruses. Alphaviruses (ss(+)RNA viruses, such as Semliki Forest virus, sindbis virus and chikungunya virus) synthesize a cap- 0 structure through a nonconventional mechanism (FIG. 3b). The Semliki Forest virus replicase protein nsP1 has N7MTase and GTase activities, the latter still incompletely characterized, and is presumably involved in the capping of viral mRNA after nsP2-mediated cleavage of the $\beta-\gamma$ phosphate bond at the $5^{\prime}$ end ${ }^{99,100}$. Although the GTase activity of nsP1 has not been fully demonstrated, GTP leads to the formation of a covalent enzyme-GMP complex, albeit only in the presence of AdoMet ${ }^{99,101}$. Accordingly, mutagenesis and in vitro assays have also revealed that the MTase catalyses the transfer of a methyl group from AdoMet to the N7 position of GTP before the formation of the covalent $\mathrm{m}^{7} \mathrm{GMP}-$ enzyme complex. The covalent link involves a conserved catalytic histidine residue that is required for the GTase reaction but not for MTase activity. Interestingly, brome mosaic virus replicase protein $1 \mathrm{~A}$, bamboo mosaic virus nsp ${ }^{102}$, tobacco mosaic virus P126 (REF. 103) and hepatitis E virus p110 (REF. 104) have properties similar to the N7MTase and GTase of alphaviruses, pointing to a common evolutionary origin for these distantly related viruses of plants and animals. Crystal structures are not yet available for any of these enzymes.

\section{Virus-mediated RNA cap snatching}

Among the ss(-)RNA viruses, those of the families Arenaviridae, Bunyaviridae and Orthomyxoviridae have a segmented RNA genome and form the order tentatively named Multinegavirales (FIG. 2). These viruses do not have a cap-synthesizing machinery. However, they have evolved to steal caps from host mRNAs in order to prime their own viral replication, in a process known as cap snatching (REFS 105-107). Cap snatching involves three steps (FIG. 3c). First, the $5^{\prime}$-methylated cap-1 or cap-2 structure of a host mRNA is bound by a specific site in the viral RdRp (or possibly the $\mathrm{N}$ protein ${ }^{108}$ ). Then, endonucleolytic cleavage of the cellular mRNA occurs several nucleotides downstream from the cap structure. Finally, this short, capped RNA is used as a primer for the synthesis of viral mRNA by the RdRp. The sequence, length and structure of the mRNA $5^{\prime}$ end that comes with the cap varies from one virus to the other. Most sequences are 15-20 nucleotides long ${ }^{106,109-112}$, but arenaviruses, nairoviruses and thogotoviruses use shorter primers ${ }^{113-116}$. Following endonucleolytic cleavage, the 'decapped' cellular mRNAs (BOX 2) are targeted to the degradation machinery, resulting in the downregulation of cellular mRNAs.

Enzymes from the cap-snatching pathway. Cap snatching was found initially in influenza viruses (ss(-)RNA viruses of the family Orthomyxoviridae), which serve as a model system for the other two viral families known to use snatching (ss(-)RNA viruses of the families Bunyaviridae and Arenaviridae), although differences in the proteins involved and the lengths of snatched sequences are expected, as shown for Thogoto virus ${ }^{117}$. The influenza virus polymerase is made of three subunits: PA, PB1 and PB2. A cap-binding domain was found in the central region of the $\mathrm{PB} 2$ subunit $^{118}$, and an endonuclease domain at the $\mathrm{N}$ terminus of the PA subunit ${ }^{119,120}$. The structures of both domains shed light on the molecular mechanisms leading to cap snatching (see below). The cap-binding domain has a novel fold, although the mode of $\mathrm{m}^{7} \mathrm{G}$ binding by aromatic stacking is similar to that used in other cap-binding proteins. By contrast, the endonuclease domain of PA has a fold that is characteristic of the two-metal-dependent $\mathrm{PD}(\mathrm{D} / \mathrm{E}) \mathrm{XK}$ nuclease superfamily but has the peculiarity of a metal-ligating histidine residue in the active site, conferring $\mathrm{Mn}^{2+}$ specificity ${ }^{121}$ (FIG. 5).

In contrast to orthomyxoviruses, both arenaviruses and bunyaviruses have a single protein (L) carrying the polymerase and cap-snatching activities. Recent studies showed that a $\mathrm{Mn}^{2+}$-dependent endonuclease that is homologous to that of orthomyxoviruses exists at the $\mathrm{N}$ terminus of arenaviral and bunyaviral L protein ${ }^{122,123}$ (FIG. 5a). Mutational analysis and cell-based replicon assays demonstrated that viral nuclease activities are essential for cap-dependent transcription of viral mRNA ${ }^{124}$. These domains have a conserved architecture and mechanism, which suggests an evolutionary link between them despite their low sequence identity (FIG. 5b).

A preliminary electron microscopy study of L protein from Machupo virus (an arenavirus) showed a central 
a

Orthomyxoviridae Endonuclease PB2 Cap-binding

Arenaviridae Bunyaviridae Endonuclease b

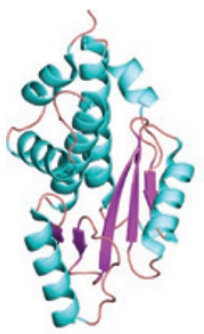

Influenza virus PA

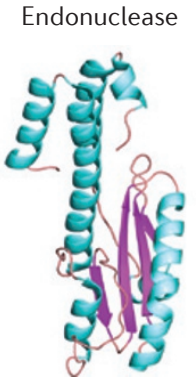

La Crosse virus L

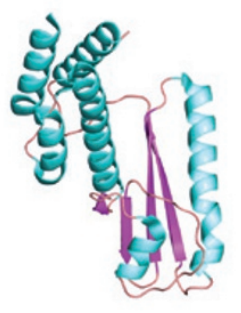

LCMV L
Cap-binding domain

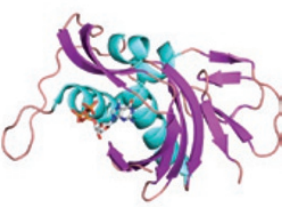

Influenza virus PB2
Figure 5 | Unconventional capping machineries. Endonucleases and cap-binding PB2. a | Domains involved in the 'cap-snatching' mechanism. The organization of cap-snatching domains of influenza viruses (single-stranded negative-sense RNA (ss(-) RNA) viruses of the family Orthomyxoviridae), and corresponding domains of distantly related viruses of the families Arenaviridae and Bunyaviridae (also ss(-)RNA viruses). Influenza virus polymerase is composed of three proteins of multiple domains: PA, PB1 and PB2. PA and PB2 are involved in cap snatching. PA carries the endonuclease domain in its amino terminus, whereas PB2 has an inner domain responsible for cap binding. Mapping of the domain organization for arenaviruses and bunyaviruses is less advanced; only the endonuclease domain is mapped to the amino terminus of $L$ protein. The cap-binding domain is not clearly identified, as it is thought to be in either $L$ protein or nucleocapsid (N or NP) protein, depending on the virus. $\mathbf{b}$ |Structures of the different endonuclease domains of viruses from the families Orthomyxoviridae (influenza viruses), Arenaviridae (lymphocytic choriomeningitis virus (LCMV)) and Bunyaviridae (La Crosse virus) (Protein Data Bank (PDB) accession codes 3 HW4, 3 JSB and 2 XI5, respectively), and of the cap-binding domain from an influenza virus (PDB accession code 2VQZ). Despite having no sequence similarities, the folds of these endonuclease domains are conserved, suggesting a convergent evolution. Structures are coloured cyan for $\alpha$-helices and pink for $\beta$-strands.

\section{Innate immunity and RNA capping}

Mammalian cells have co-evolved with viruses and have developed several mechanisms to detect a viral infection (such as detecting uncapped or partially capped RNA, VPg-RNA, and so on) and induce an antiviral response in neighbouring cells ${ }^{130-132}$. This innate immunity is based on a small number of receptors called pattern recognition receptors (PRRs), which discriminate self from non-self components. Non-self detection depends on the recognition of a limited set of pathogenassociated molecular patterns (PAMPs), which are molecules or components that are characteristic of infectious agents, such as viral nucleic acids ${ }^{133}$. The presence of non-self nucleic acids is detected through sensors such as Toll-like receptors (TLRs), which recognize DNA or RNA in intracellular compartments that do not usually contain these molecules. Moreover, several PRRs sense the presence of foreign nucleic acids directly in the cytoplasm - namely, the NOD-like receptors (NLRs) and the retinoic acid-inducible gene (RIG)-like receptors (RLRs) ${ }^{134}$. As host cell RNA is present in the cytosol, PRRs sense uncommon RNA structures that are present in infected cells, such as dsRNA, RNA presenting a $5^{\prime}$-triphosphate, RNA with an incompletely methylated cap structure (cap-0, for mammalian standards) or RNA bearing a protein covalently attached to the $5^{\prime}$ end (such as VPg). The detection of PAMPs by PRRs triggers intracellular signalling events that mainly induce the production of type I interferon (IFN), interleukin-1 (IL-1) and pro-inflammatory cytokines, as well as the establishment of a cellular antiviral state in order to limit viral propagation ${ }^{135,136}$ (FIG. 6).

Among the PRRs, the TLR family is the best studied innate immunity sensor family. TLRs are transmembrane proteins that are mainly expressed in immune cells, such as macrophages and dendritic cells. They are localized in endosomal compartments or at the cell surface. TLRs contain a leucine-rich repeat motif that recognizes PAMPs, and a Toll-IL-1 receptor (TIR) domain is present in the cytoplasmic part of the protein, ensuring signal transduction through TIR domain interaction with the TIR domains of cytoplasmic adaptor proteins such as MYD88 and TRIF (also known as TICAM1). TLR3, TLR7 and TLR8 are activated by different kinds of RNAs. TLR3 detects dsRNA, whereas TLR7 and TLR8 recognize ssRNA carrying a $5^{\prime}$-triphosphate or a cap-0 structure ${ }^{137}$.

In contrast to TLRs, the NLRs and RLRs are localized in the cell cytoplasm ${ }^{132}$ and detect the presence of intracellular invaders. Among the RLRs, RIG-I (also known as DDX58) and MDA5 (also known as IFIH1) seem to discriminate non-self RNA from self RNA on the basis of the RNA $5^{\prime}$ end ${ }^{134}$. Metazoan self RNA presents a $5^{\prime}$ cap-1 or cap- 2 structure. RIG-I protein is specialized in the detection of $5^{\prime}$-triphosphate RNA, whereas MDA5 senses the presence of RNA with a cap-0 structure or linked to a protein such as $\mathrm{VPg}^{138}$. RIG-I and MDA5 consist of two N-terminal caspase-recruitment domains (CARDs), a central DEXH box-containing RNA helicase-ATPase domain and a C-terminal regulatory domain $(\mathrm{CTD})^{139}$. It is likely that, in the absence 


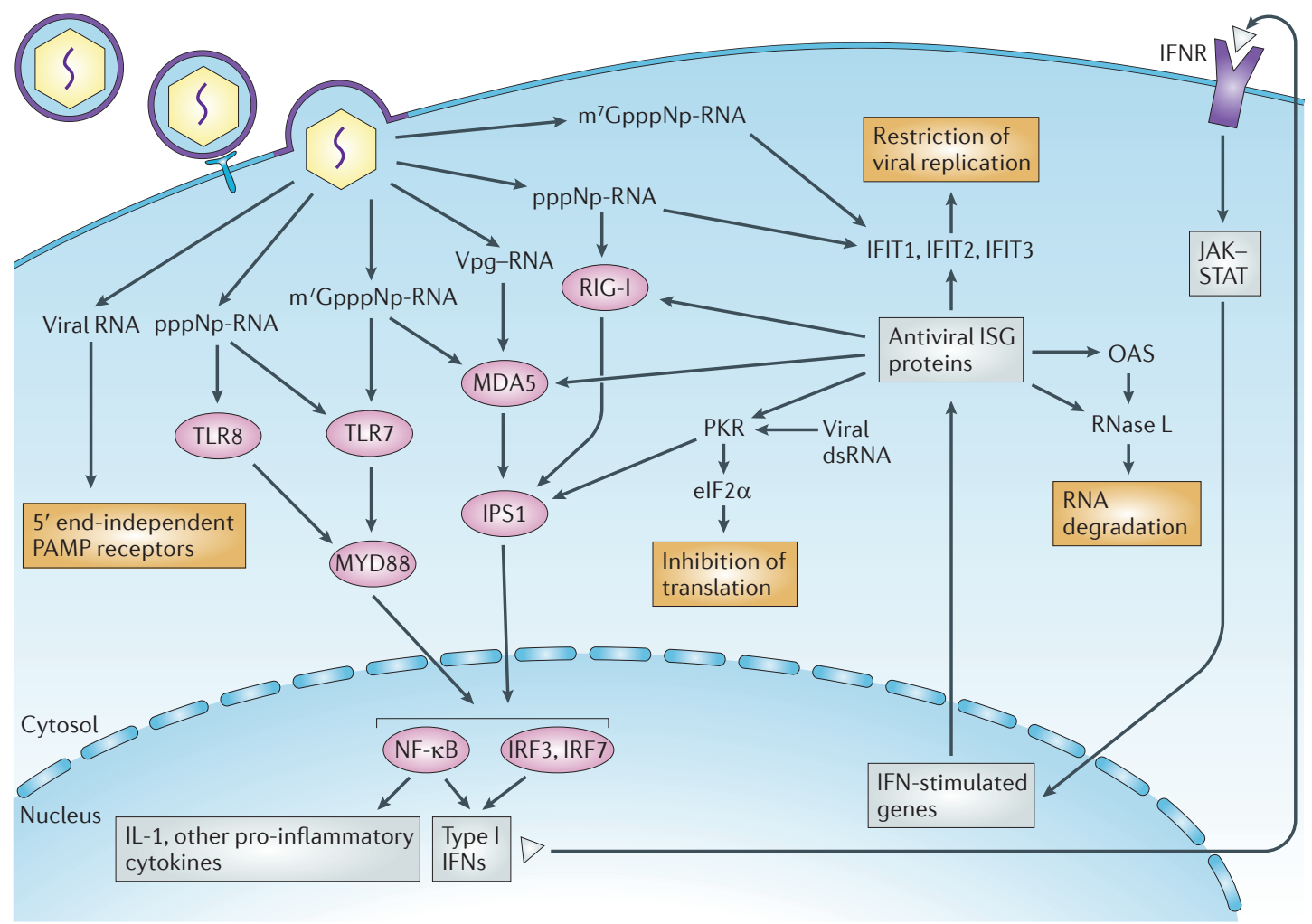

Figure 6 | Sensing of viral RNA by the innate immune system. A virus that infects a cell releases viral RNA into the host cytoplasm or endosomes in one of four forms: RNA protected by a 5' cap-1 structure (7-methyl-Gppp-2'-O-methyl-

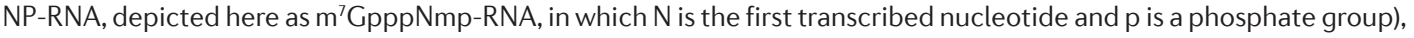
5'-triphosphate RNA (pppNp-RNA), RNA linked to VPg or RNA carrying a 5' cap-0 structure (m GpppNp-RNA). Cap-1 RNA is recognized by pattern recognition receptors (PRRs) such as Toll-like receptors (TLRs), retinoic acid-inducible gene (RIG)-like receptors (RLRs) and NOD-like receptors (NLRs), which in this case recognize, for example, double-stranded RNA (dsRNA). In absence of a cap structure, the 5'-triphosphate of RNA is sensed by the RLR RIG-I, and the VPg-RNA and cap-0 RNA are recognized by another RLR, MDA5. Both RIG-I and MDA5 recruit the mitochondrial-anchored protein interferon- $\beta$ (IFN $\beta$ ) promoter stimulator 1 (IPS1), which in turn recruits appropriate inhibitor of NF- $\kappa B$ kinase (IKK) proteins to activate nuclear factor- $\mathrm{KB}(\mathrm{NF}-\kappa \mathrm{B})$ and IFN regulatory factors (IRFs). This results in the induction of type I IFNs and the production of pro-inflammatory cytokines such as interleukin-1 (IL-1). The endosomal receptor TLR7 can recognize cap-0 RNA and 5'-triphosphate RNA, whereas TLR8 recognizes only 5'-triphosphate RNA. TLR7 recruits the adaptor protein MYD88 through a Toll-IL-1 receptor (TIR)-TIR domain interaction, leading to the activation of NF- $\mathrm{kB}$ and IRF3 or IRF7; this results in the induction of type I IFNs and the production of IL-1 and other pro-inflammatory cytokines. Antiviral restriction factors are also stimulated by type I IFN receptor (IFNR) and are known to inhibit the replication of RNA viruses carrying non-capped genomes. Autocrine and paracrine IFN binds IFNR and initiates the JAK-STAT (Janus kinase-signal transducer and activator of transcription) signalling cascade. Among hundreds of proteins encoded by IFN-stimulated genes (ISGs), some antiviral proteins specifically target uncapped viral RNA. The IFIT (IFN-induced protein with tetratricopeptide repeats) proteins specifically sequester $5^{\prime}$-triphosphate RNA (IFIT1) or cap-0 RNA (IFIT2). Protein kinase, RNA activated (PKR) recognizes 5'-triphosphate RNA through an amino-terminal dsRNA-binding domain composed of two binding motifs. Activated PKR phosphorylates eukaryotic translation initiation factor $2 a$ (elF2 $\alpha$ ) through its kinase domain and blocks protein translation. RNase L is stimulated by $2^{\prime}, 5^{\prime}$ oligo(A) oligonucleotides synthesized by oligoadenylyl synthases (OASs) and is also involved in the degradation of capped and non-capped viral RNA.

of ligands, the CARDs of RIG-I are auto-inhibited by other domains of the protein. For RIG-like proteins, nucleic acid binding to the RNA-binding site of the CTD induces a conformational change resulting in interaction of the CARD with the signalling adaptor molecule IFN $\beta$ promoter stimulator 1 (IPS1; also known as CARDIF, MAVS or VISA). IPS1 recruits a signalling complex in order to activate transcription factors such as interferon regulatory factor 3 (IRF3) and nuclear factor- $\kappa \mathrm{B}$ $(\mathrm{NF}-\mathrm{\kappa B})$, leading to the expression of IFN $\beta$ and other proteins that drive the antiviral response (FIG. 6).
The molecular basis for RNA recognition with and without $5^{\prime}$ end modification and/or overhanging nucleotides was analysed for RIG-I and MDA5. Initial studies indicated that RIG-I specifically recognizes $5^{\prime}$-triphosphate-containing ssRNA ${ }^{140}$. It was later found that RIG-I requires base-paired structures in conjunction with a $5^{\prime}$-triphosphate to trigger an antiviral response $\mathrm{e}^{141}$. The molecular basis of the specific interaction between the RIG-I CTD and 5'-triphosphate dsRNA was deciphered by a crystallographical study of the CTD in complex with RNA ${ }^{142}$. This study revealed 
Viral antigenomes

Viral RNAs that are complementary strands to

the genome. The antigenome strand is used as a matrix for the synthesis of new viral genomes, and of viral mRNAs in the case of positive-sense RNA viruses. that 5'-triphosphate dsRNA binds to the CTD of RIG-I more tightly than its single-stranded counterpart. The $5^{\prime}$-triphosphate is sequestered in a lysine-rich cleft of the CTD, with a phenylalanine residue stacked to the terminal base pair. Interestingly, $5^{\prime}$-triphosphate dsRNA methylated at the $2^{\prime}-O$ position of its first or second nucleotide is expected to create a steric conflict with the CTD of RIG-I. Accordingly, it does not stimulate the RIG-I pathway.

In contrast to RIG-I, MDA5 is thought to recognize either a viral RNA 5' end carrying structures that are distinct from a $5^{\prime}$-triphosphate or longer, structured (mesh) RNA that would be generated during the viral life cycle. Indeed, MDA5 was reported to sense both dsRNA and ssRNA bearing a $5^{\prime}$ cap-0 (REFS 138,143) or linked to VPg. Structural analysis of the MDA5 CTD indicated that its global fold is similar to that of the RIG-I CTD ${ }^{144}$, with amino acid differences in the domains involved in the recognition of the RNA $5^{\prime}$ end.

The involvement of MDA5 and TLR7 in the detection of cap-0-containing RNA was recently characterized in the antiviral response observed for a coronavirus mutant lacking $2^{\prime}$ OMTase activity ${ }^{138}$. The replication of this mutant virus was dramatically impaired in infected mice. However, the replication of this virus was restored in MDA5-, TLR7- or type I IFN receptor (IFNR)deficient mice. It has also been suggested that other interferon-stimulated genes (ISGs) restrict the replication of $2^{\prime}$ OMTase-deficient viruses ${ }^{145}$. Accordingly, IFIT1 (IFN-induced protein with tetratricopeptide repeats 1; also known as IFI56) and IFIT2 (also known as IFI54) were reported to limit the replication of West Nile virus (an ss(+)RNA virus of the genus Flavivirus), vaccinia virus (a dsDNA virus of the family Poxviridae) and murine hepatitis virus (an ss(+)RNA virus of the family Coronaviridae) lacking $2^{\prime}$ OMTase activity ${ }^{145}$. IFIT1 was recently shown to bind and sequester $5^{\prime}$-triphosphate RNA into a multiprotein complex containing IFIT2 and IFIT3 (also known as IFI60) in order to exert its antiviral effect ${ }^{146}$. Therefore, it is likely that $2^{\prime}$-O-methylation of the RNA cap promotes escape from the host innate antiviral response through avoidance of IFIT-mediated suppression.

\section{Conclusions}

Since the discovery of 'blocked and methylated' mRNA ends nearly 40 years ago, viruses have played an essential part in deciphering the process of mRNA capping, as well as its relationship with various cellular processes such as transcription, translation and innate immunity. Viral RNA capping is a field that still has a lot of uncharted territory: whether the RNA $5^{\prime}$ ends are protected or not is still unknown for many neglected viral families, and the GTase resists identification even for some studied human pathogens (for example, the ss $(+$ ) RNA viruses of the order Nidovirales).

It is likely that, during their co-evolution with their hosts, viruses evolved different adaptation strategies to protect their RNA transcripts. The diversity of mechanisms expressed in nature to add a cap to an RNA $5^{\prime}$ end is larger than that described here. Future research will also aim to elucidate how the fine-tuning between host-mediated decapping of viral RNA, virusmediated capping of viral RNA and host innate immunity is performed. Moreover, it has been shown that viral antigenomes are not capped ${ }^{147}$, an observation that has now been extended to many different viruses. Thus, 'no capping' signals probably exist. In addition, the abundance of template RNAs must certainly need to be finely regulated for optimal viral replication. The corresponding signals and regulations are largely unknown.

Finally, owing to its spectacular mechanistic diversity, RNA capping is an attractive field for the design of antiviral drugs. Several molecules have been proposed to act directly or indirectly on viral RNA capping. Ribavirin is a broad-spectrum antiviral agent that is active against several viruses that add or snatch RNA caps, and its pleiotropic mechanism includes targeting the RNA-capping machinery ${ }^{148,149}$. So far, efforts to design MTase inhibitors have used the AdoMet and AdoHcy ( $S$-adenosyl-Lhomocysteine) backbone to synthesize analogues that are specific to viral enzymes ${ }^{150}$. The increasing knowledge about active-site differences between cellular and viral MTases is expected to provide antiviral selectivity. Last, inhibitors of cap-snatching endonuclease have long been known ${ }^{119,151-153}$. Recently published crystal structures $^{119,122,123}$ of their targets should inform antiviral-drug design projects.
1. Shatkin, A. Capping of eucaryotic mRNAs. Cell 9, 645 (1976).

2. Darnell, J. E. Jr. Transcription units for mRNA production in eukaryotic cells and their DNA viruses. Prog. Nucleic Acid Res. Mol. Biol. 22, 327-353 (1979).

3. Filipowicz, W. et al. A protein binding the methylated $5^{\prime}$-terminal sequence, $\mathrm{m}^{7} \mathrm{GpppN}$, of eukaryotic messenger RNA. Proc. Natl Acad. Sci. USA 73 1559-1563 (1976).

4. Schibler, U. \& Perry, R. P. The 5'-termini of heterogeneous nuclear RNA: a comparison among molecules of different sizes and ages. Nucleic Acids Res. 4, 4133-4149 (1977).

5. Liu, H. \& Kiledjian, M. Decapping the message: beginning or an end. Biochem. Soc. Trans. 34, 35-38 (2006).

6. Nallagatla, S. R., Toroney, R. \& Bevilacqua, P. C. A brilliant disguise for self RNA: 5'-end and internal modifications of primary transcripts suppress elements of innate immunity. RNA Biol. 5, 140-144 (2008).
Rehwinkel, J. et al. RIG-I detects viral genomic RNA during negative-strand RNA virus infection. Cell 140 397-408 (2010).

8. Furuichi, Y. \& Shatkin, A. J. Viral and cellular mRNA capping: past and prospects. Adv. Vir. Res. $\mathbf{5 5}$ 135-184 (2000).

A historical and chronological perspective on the discovery of RNA capping and the structure of the RNA cap.

9. Furuichi, Y., Muthukrishnan, S. \& Shatkin, A. J. $5^{\prime}$-Terminal $\mathrm{m}^{-7} \mathrm{G}\left(5^{\prime}\right) \mathrm{ppp}\left(5^{\prime}\right) \mathrm{G}^{\mathrm{m}} \mathrm{p}$ in vivo: identification in reovirus genome RNA. Proc. NatI Acad. Sci. USA 72, 742-745 (1975).

10. Shatkin, A. J. Methylated messenger RNA synthesis in vitro by purified reovirus. Proc. Natl Acad. Sci. USA 71, 3204-3207 (1974).

11. Wei, C. M. \& Moss, B. Methylated nucleotides block 5 '-terminus of vaccinia virus messenger RNA. Proc Natl Acad. Sci. USA 72, 318-322 (1975).

12. Shatkin, A. J. \& Both, G. W. Reovirus mRNA: transcription translation. Cell 7, 305-313 (1976).
13. Furuichi, Y., Morgan, M., Muthukrishnan, S. \& Shatkin, A. J. Reovirus messenger RNA contains a methylated, blocked $5^{\prime}$-terminal structure: $\mathrm{m}^{7} \mathrm{G}\left(5^{\prime}\right)$ ppp $\left(5^{\prime}\right) \mathrm{G}^{\mathrm{m}} \mathrm{pCp}$-. Proc. Natl Acad. Sci. USA 72 362-366 (1975)

14. Muthukrishnan, S., Both, G. W., Furuichi, Y. \& Shatkin, A. J. 5'-Terminal 7-methylguanosine in eukaryotic mRNA is required for translation. Nature 255, 33-37 (1975).

15. Marcotrigiano, J., Gingras, A. C., Sonenberg, N. \& Burley, S. K. Cocrystal structure of the messenger RNA 5 ' cap-binding protein (elF4E) bound to 7-methyl-GDP. Cell 89, 951-961 (1987).

16. Perry, K. L., Watkins, K. P. \& Agabian, N. Trypanosome mRNAs have unusual "cap 4" structures acquired by addition of a spliced leader. Proc. Natl Acad. Sci. USA 84, 8190-8194 (1987)

17. Beelman, C. A. \& Parker, R. Degradation of mRNA in eukaryotes. Cell 81, 179-183 (1995).

18. Houseley, J. \& Tollervey, D. The many pathways of RNA degradation. Cell 136, 763-776 (2009). 
19. Otsuka, Y., Kedersha, N. L. \& Schoenberg, D. R. Identification of a cytoplasmic complex that adds a cap onto 5'-monophosphate RNA. Mol. Cell. Biol. 29 2155-2167 (2009).

20. Schoenberg, D. R. \& Maquat, L. E. Re-capping the message. Trends Biochem. Sci. 34, 435-442 (2009).

21. Goodfellow, I. et al. Calicivirus translation initiation requires an interaction between $\mathrm{VPg}$ and elF4E. EMBO Rep. 6, 968-972 (2005).

22. Ambros, V., Pettersson, R. F. \& Baltimore, D. An enzymatic activity in uninfected cells that cleaves the linkage between poliovirion RNA and the $5^{\prime}$ terminal protein. Cell 15, 1439-1446 (1978)

23. Ambros, V. \& Baltimore, D. Purification and properties of a HeLa cell enzyme able to remove the 5'-terminal protein from poliovirus RNA. J. Biol. Chem. 255 6739-6744 (1980).

24. Cong, P. \& Shuman, S. Mutational analysis of mRNA capping enzyme identifies amino acids involved in GTP binding, enzyme-guanylate formation, and GMP transfer to RNA. Mol. Cell. Biol. 15, 6222-6231 (1995).

25. De la Peña, M., Kyrieleis, O. J. P. \& Cusack, S Structural insights into the mechanism and evolution of the vaccinia virus mRNA cap N7 methyl-transferase. EMBO J. 26, 4913-4925 (2007).

Work showing activation of the MTase domain of D1 in complex with the D12 subunit at atomic resolution.

26. Mao, X. \& Shuman, S. Intrinsic RNA (guanine-7) methyltransferase activity of the vaccinia virus capping enzyme D1 subunit is stimulated by the D1 2 subunit. Identification of amino acid residues in the D1 protein required for subunit association and methyl group transfer. J. Biol. Chem. 269, 24472-24479 (1994). The discovery and molecular basis of the activation of a viral MTase by a protein cofactor.

27. Schnierle, B. S., Gershon, P. D. \& Moss, B. Cap-specific mRNA (nucleoside- $O^{2}$-)-methyltransferase and poly(A) polymerase stimulatory activities of vaccinia virus are mediated by a single protein. Proc. Natl Acad. Sci. USA 89, 2897-2901 (1992).

28. Benarroch, D. et al. The RNA helicase, nucleotide 5'-triphosphatase, and RNA 5'-triphosphatase activities of Dengue virus protein NS3 are $\mathrm{Mg}^{2+}$ dependent and require a functional Walker $\mathrm{B}$ motif in the helicase catalytic core. Virology 328, 208-218 (2004).

29. Myette, J. R. \& Niles, E. G. Characterization of the vaccinia virus RNA 5'-triphosphatase and nucleotide triphosphate phosphohydrolase activities demonstrate that both activities are carried out at the same active site. J. Biol. Chem. 271, 11945-11952 (1996)

30. Vasquez-Del Carpio, R., Gonzalez-Nilo, F. D., Riadi, G., Taraporewala, Z. F. \& Patton, J. T. Histidine triad-like motif of the rotavirus NSP2 octamer mediates both RTPase and NTPase activities. J. Mol. Biol. 362, 539-554 (2006)

31. Jayaram, H., Taraporewala, Z., Patton, J. T. \& Prasad, B. V. V. Rotavirus protein involved in genome replication and packaging exhibits a HIT-like fold Nature 417, 311-315 (2002) The first crystal structure of an RTPase with a HIT-like fold.

32. Taraporewala, Z, Chen, D. \& Patton, J. T. Multimers formed by the rotavirus nonstructural protein NSP2 bind to RNA and have nucleoside triphosphatase activity. J. Virol. 73, 9934-9943 (1999).

33. Taraporewala, Z. F. \& Patton, J. T. Identification and characterization of the helix-destabilizing activity of rotavirus nonstructural protein NSP2. J. Virol. 75, 4519-4527 (2001).

34. Benarroch, D., Smith, P. \& Shuman, S. Characterization of a trifunctional mimivirus mRNA capping enzyme and crystal structure of the RNA triphosphatase domain. Structure 16, 501-512 (2008).

35. Gu, M. \& Lima, C. D. Processing the message: structural insights into capping and decapping mRNA. Curr. Opin. Struc. Biol. 15, 99-106 (2005).

36. Lima, C. D., Wang, L. K. \& Shuman, S. Structure and mechanism of yeast RNA triphosphatase: an essential component of the mRNA capping apparatus. Cell 99, 533-543 (1999).

The first crystal structure of an RTPase involved in RNA capping.

37. Shuman, S. Structure, mechanism, and evolution of the mRNA capping apparatus. Prog. Nucleic Acid Res. Mol. Biol. 66, 1-40 (2001).

38. Lehman, K., Schwer, B., Ho, C. K., Rouzankina, I. \& Shuman, S. A conserved domain of yeast RNA triphosphatase flanking the catalytic core regulates self-association and interaction with the guanylyltransferase component of the mRNA capping apparatus. J. Biol. Chem. 274, 22668-22678 (1999).

39. Gorbalenya, A. E. \& Koonin, E. V. One more conserved sequence motif in helicases. Nucleic Acids Res. 16, 7734 (1988)

40. Gorbalenya, A. E. \& Koonin, E. V. Viral proteins containing the purine NTP-binding sequence pattern. Nucleic Acids Res. 17, 8413-8440 (1989).

41. Gorbalenya, A. E., Koonin, E. V., Donchenko, A. P. \& Blinov, V. M. Two related superfamilies of putative helicases involved in replication, recombination, repair and expression of DNA and RNA genomes. Nucleic Acids Res. 17, 4713-4730 (1989).

42. Luo, D. et al. Insights into RNA unwinding and ATP hydrolysis by the flavivirus NS3 protein. EMBO J. 27 3209-3219 (2008)

43. Gu, M. \& Rice, C. M. Three conformational snapshots of the hepatitis $C$ virus NS3 helicase reveal a ratchet translocation mechanism. Proc. Natl Acad. Sci. USA 107, 521-528 (2010)

44. Lescar, J. et al. Towards the design of antiviral inhibitors against flaviviruses: the case for the multifunctional NS3 protein from Dengue virus as a target. Antiviral Res. 80, 94-101 (2008).

45. Luo, D. et al. Flexibility between the protease and helicase domains of the dengue virus NS3 protein conferred by the linker region and its functional implications. J Biol. Chem. 285, 18817-18827 (2010).

46. Luo, D. et al. Crystal structure of the NS3 proteasehelicase from dengue virus. J. Virol. 82, 173-183 (2008)

47. Sampath, A. et al. Structure-based mutational analysis of the NS3 helicase from dengue virus. J. Virol. $\mathbf{8 0}$ 6686-6690 (2006)

48. Balistreri, G., Caldentey, J., Kääriäinen, L. \& Ahola, T. Enzymatic defects of the nsP2 proteins of Semliki Forest virus temperature-sensitive mutants. $J$ Virol. 81, 2849-2860 (2007).

49. Changela, A., Ho, C. K., Martins, A., Shuman, S. \& Mondragón, A. Structure and mechanism of the RNA triphosphatase component of mammalian mRNA capping enzyme. EMBO J. 20, 2575-2586 (2001).

50. Takagi, T., Moore, C. R., Diehn, F. \& Buratowski, S. An RNA 5'-triphosphatase related to the protein tyrosine phosphatases. Cell 89, 867-873 (1997).

51. Shuman, S. \& Lima, C. D. The polynucleotide ligase and RNA capping enzyme superfamily of covalent nucleotidyltransferases. Curr. Opin. Struct. Biol. 14 757-764 (2004).

52. Wang, S. P., Deng, L., Ho, C. K. \& Shuman, S. Phylogeny of mRNA capping enzymes. Proc. Natl Acad. Sci. USA 94, 9573-9578 (1997).

53. Cong, P. \& Shuman, S. Covalent catalysis in nucleotidyl transfer. A KTDG motif essential for enzyme-GMP complex formation by mRNA capping enzyme is conserved at the active sites of RNA and DNA ligases. J. Biol. Chem. 268, 7256-7260 (1993).

54. Niles, E. G. \& Christen, L. Identification of the vaccinia virus mRNA guanyltransferase active site lysine. J. Biol. Chem. 268, 24986-24989 (1993).

55. Shuman, S. \& Hurwitz, J. Mechanism of mRNA capping by vaccinia virus guanylyltransferase: characterization of an enzyme-guanylate intermediate. Proc. Natl Acad. Sci. USA 78, 187-191 (1981).

56. Schwer, B. \& Shuman, S. Mutational analysis of yeast mRNA capping enzyme. Proc. Natl Acad. Sci. USA 91 , 4328-4332 (1994)

57. Lindahl, T. \& Barnes, D. E. Mammalian DNA ligases. Annu. Rev. Biochem. 61, 251-281 (1992).

58. Chu, C. et al. Structure of the guanylyltransferase domain of human mRNA capping enzyme. Proc. Nat Acad. Sci. USA 108, 10104-10108 (2011).

59. Fabrega, C., Shen, V., Shuman, S. \& Lima, C. D. Structure of an mRNA capping enzyme bound to the phosphorylated carboxy-terminal domain of RNA polymerase II. Mol Cell 11, 1549-1561 (2003).

60. Håkansson, K., Doherty, A. J., Shuman, S. \& Wigley, D. B. X-ray crystallography reveals a large conformational change during guanyl transfer by mRNA capping enzymes. Cell 89, 545-553 (1997). A study that deciphers the GTase reaction, with corresponding crystal structure snapshots.

61. Håkansson, K. \& Wigley, D. B. Structure of a complex between a cap analogue and mRNA guanylyl transferase demonstrates the structural chemistry of RNA capping. Proc. Natl Acad. Sci. USA 95. 1505-1510 (1998)
62 Reinisch, K. M., Nibert, M. L. \& Harrison, S. C. Structure of the reovirus core at 3.6 A resolution. Nature 404, 960-967 (2000) A report detailing the complete orthoreoviral RNA cap assembly line at atomic resolution.

63. Sutton, G., Grimes, J. M., Stuart, D. I. \& Roy, P. Bluetongue virus VP4 is an RNA-capping assembly line. Nature Struct. Mol. Biol. 14, 449-451 (2007).

64. Cheng, L et al. Atomic model of a cypovirus built from cryo-EM structure provides insight into the mechanism of mRNA capping. Proc. Natl Acad. Sci. USA 108 , 1373-1378 (2011)

65. Kim, J., Tao, Y., Reinisch, K. M., Harrison, S. C. \& Nibert, M. L. Orthoreovirus and Aquareovirus core proteins: conserved enzymatic surfaces, but not protein-protein interfaces. Virus Res. 101, 15-28 (2004).

66. Luongo, C. L., Reinisch, K. M., Harrison, S. C. \& Nibert, M. L. Identification of the guanylyltransferase region and active site in reovirus mRNA capping protein $\lambda 2$. J. Biol. Chem. 275, 2804-2810 (2000).

67. Issur, M. et al. The flavivirus NS5 protein is a true RNA guanylyltransferase that catalyzes a two-step reaction to form the RNA cap structure. RNA 15 2340-2350 (2009).

68. Bisaillon, M. \& Lemay, G. Viral and cellular enzymes involved in synthesis of mRNA cap structure. Virology 236, 1-7 (1997).

69. Egloff, M. P., Benarroch, D., Selisko, B., Romette, J. L. $\&$ Canard, B. An RNA cap (nucleoside-2'-O-)methyltransferase in the flavivirus RNA polymerase NS5: crystal structure and functional characterization. EMBO J. 21, 2757-2768 (2002).

Characterization of the first ( +)RNA virus MTase at atomic resolution.

70. Martin, J. L. \& McMillan, F. M. SAM (dependent) I AM: the $S$-adenosylmethionine-dependent methyltransferase fold. Curr. Opin. Struct. Biol. 12, 783-793 (2002)

71. Schubert, H. Many paths to methyltransfer: a chronicle of convergence. Trends Biochem. Sci. 28, 329-335 (2003).

72. Decroly, E. et al. Crystal structure and functional analysis of the SARS-coronavirus RNA cap 2'-O-methyltransferase nsp 10/nsp 16 complex. PLoS Pathog. 7, e1002059 (2011).

73. Egloff, M. P. et al. Structural and functional analysis of methylation and 5'-RNA sequence requirements of short capped RNAs by the methyltransferase domain of dengue virus NS5. J. Mol. Biol. 372, 723-736 (2007).

74. Dong, H., Ren, S., Li, H. \& Shi, P. Y. Separate molecules of West Nile virus methyltransferase can independently catalyze the $\mathrm{N} 7$ and 2'-O methylations of viral RNA cap. Virology 377, 1-6 (2008).

75. Ray, D. et al. West Nile virus 5 '-cap structure is formed by sequential guanine $\mathrm{N}-7$ and ribose 2 '-O methylations by nonstructural protein 5. J. Virol. 80 , 8362-8370 (2006).

76. Fabrega, C., Hausmann, S., Shen, V., Shuman, S. \& Lima, C. D. Structure and mechanism of mRNA cap (guanine-N7) methyltransferase. Mol. Cell 13, 77-89 (2004).

77. Bujnicki, J. M. \& Rychlewski, L. Reassignment of specificities of two cap methyltransferase domains in the reovirus $\lambda 2$ protein. Genome Biol. 2 RESEARCH0038.1-RESEARCH0038.6 (2001).

78 Ferron, F., Longhi, S., Henrissat, B \& Canard, B. Viral RNA-polymerases - a predicted 2'-O-ribose methyltransferase domain shared by all Mononegavirales. Trends Biochem. Sci. 27, 222-224 (2002).

79. Hodel, A. E., Gershon, P. D., Shi, X. \& Quiocho, F. A. The $1.85 \AA$ structure of vaccinia protein VP39: a bifunctional enzyme that participates in the modification of both mRNA ends. Cell 85, 247-256 (1996).

The first crystal structure of a viral enzyme involved in RNA capping.

80. Lockless, S. W., Cheng, H. T., Hodel, A. E., Quiocho, F. A \& Gershon, P. D. Recognition of capped RNA substrates by VP39, the vaccinia virus-encoded mRNA cap-specific 2'-O-methyltransferase. Biochemistry 37 8564-8574 (1998).

81. Li, C., Xia, Y., Gao, X. \& Gershon, P. D. Mechanism of RNA 2'-O-methylation: evidence that the catalytic lysine acts to steer rather than deprotonate the target nucleophile. Biochemistry 43, 5680-5687 (2004). 
82 Hodel, A. E. Gershon, P. D. \& Quiocho, F. A. Structural basis for sequence-nonspecific recognition of 5 -capped mRNA by a cap-modifying enzyme. Mol. Cell 1, 443-447 (1998).

An article discussing the molecular basis of N7-methylated-cap selectivity and detailing the first RNA cap protein crystal structure.

83. Bouvet, M. et al. In vitro reconstitution of SARScoronavirus mRNA cap methylation. PLoS Pathog. 6 , e1000863 (2010).

84. Chen, Y. et al. Functional screen reveals SARS coronavirus nonstructural protein nsp 14 as a novel cap N7 methyltransferase. Proc. Natl Acad. Sci USA 106, 3484-3489 (2009).

85. Decroly, E. et al. Coronavirus nonstructural protein 16 is a cap-0 binding enzyme possessing (nucleoside- $2^{\prime} \mathrm{O}$ ) methyltransferase activity. J. Virol. 82, 8071-8084 (2008).

86. Ramadevi, N., Burroughs, N. J., Mertens, P. P., Jones, I. M. \& Roy, P. Capping and methylation of mRNA by purified recombinant VP4 protein of bluetongue virus. Proc. Natl Acad. Sci. USA 95, 13537-13542 (1998)

87. Ramadevi, N. \& Roy, P. Bluetongue virus core protein VP4 has nucleoside triphosphate phosphohydrolase activity. J. Gen. Virol. 79, 2475-2480 (1998).

88. Abraham, G., Rhodes, D. P. \& Banerjee, A. K. The $5^{\prime}$ terminal structure of the methylated mRNA synthesized in vitro by vesicular stomatitis virus. Cell 5, 51-58 (1975)

89. Abraham, G., Rhodes, D. P. \& Banerjee, A. K Novel initiation of RNA synthesis in vitro by vesicular stomatitis virus. Nature 255, 37-40 (1975).

90. Gupta, K. C. \& Roy, P. Alternate capping mechanisms for transcription of spring viremia of carp virus: evidence for independent mRNA initiation. J. Virol. 33, 292-303 (1980)

91. Barik, S. The structure of the $5^{\prime}$ terminal cap of the respiratory syncytial virus mRNA. J. Gen. Virol. 74 485-490 (1993)

92. Ogino, T. \& Banerjee, A. K. The HR motif in the RNAdependent RNA polymerase L protein of Chandipura virus is required for unconventional mRNA-capping activity. J. Gen. Virol. 91, 1311-1314 (2010).

93. Ogino, T. \& Banerjee, A. K. Unconventional mechanism of mRNA capping by the RNA-dependent RNA polymerase of vesicular stomatitis virus. Mol. Cell 25, 85-97 (2007)

The elucidation of the unconventional RNA-capping pathway of (-)RNA viruses.

94. Bujnicki, J. M. \& Rychlewski, L. In silico identification, structure prediction and phylogenetic analysis of the 2'-O-ribose (cap 1) methyltransferase domain in the large structural protein of ssRNA negative-strand viruses. Protein Eng. 15, 101-108 (2002).

95. Li, J., Fontaine-Rodriguez, E. C. \& Whelan, S. P. Amino acid residues within conserved domain VI of the vesicular stomatitis virus large polymerase protein essential for mRNA cap methyltransferase activity. J. Virol. 79, 13373-13384 (2005)

96. Rahmeh, A. A., Li, J., Kranzusch, P. J. \& Whelan, S. P. J. Ribose 2'-O methylation of the vesicular stomatitis virus mRNA cap precedes and facilitates subsequent guanine- $\mathrm{N}-7$ methylation by the large polymerase protein. J. Virol. 83, 11043-11050 (2009). A paper with significant implications for understanding the mechanisms of unconventional capping in VSV. The authors show the sequence requirements for methylation and the chain of events that characterize the mechanism.

97. Testa, D. \& Banerjee, A. K. Two methyltransferase activities in the purified virions of vesicular stomatitis virus. J. Virol. 24, 786-793 (1977)

98. Tekes, G., Rahmeh, A. A. \& Whelan, S. P. A freeze frame view of vesicular stomatitis virus transcription defines a minimal length of RNA for 5' processing. PLoS Pathog. 7, e1002073 (2011).

99. Ahola, T. \& Kääriäinen, L. Reaction in alphavirus mRNA capping: formation of a covalent complex of nonstructural protein nsP1 with 7-methyl-GMP. Proc Natl Acad. Sci. USA 92, 507-511 (1995).

The discovery of the unconventional RNA-capping pathway of alphaviruses, in which guanine-N7 methylation of GTP precedes $\mathrm{m}^{7} \mathrm{GMP}$ transfer onto the putative GTase, nsP1.

100. Vasiljeva, L., Merits, A., Auvinen, P. \& Kääriäinen, L. Identification of a novel function of the alphavirus capping apparatus. RNA 5'-triphosphatase activity of Nsp2. J. Biol. Chem. 275, 17281-17287 (2000).

101. Ahola, T., Laakkonen, P., Vihinen, H. \& Kääriäinen, L. Critical residues of Semliki Forest virus RNA capping enzyme involved in methyltransferase and guanylyltransferase-like activities. J. Virol. 71 392-397 (1997)

102. Li, Y. I., Chen, Y. J., Hsu, Y. H. \& Meng, M. Characterization of the AdoMet-dependent guanylyltransferase activity that is associated with the $\mathrm{N}$ terminus of bamboo mosaic virus replicase. J. Virol. 75, 782-788 (2001).

103. Merits, A. et al. Virus-specific capping of tobacco mosaic virus RNA: methylation of GTP prior to formation of covalent complex p126-m7GMP. FEBS Lett. 455, 45-48 (1999).

104. Magden, J. et al. Virus-specific mRNA capping enzyme encoded by hepatitis E virus. J. Virol. 75, 6249-6255 (2001).

105. Bouloy, M., Plotch, S. J. \& Krug, R. M. Globin mRNAs are primers for the transcription of influenza viral RNA in vitro. Proc. Natl Acad. Sci. USA 75, 4886-4890 (1978)

106. Caton, A. J. \& Robertson, S. Structure of the hostderived sequences present at the $5^{\prime}$ ends of influenza virus mRNA. Nucleic Acids Res. 8, 2591-2603 (1980).

107. Plotch, S. J., Bouloy, M. \& Krug, R. M. Transfer of 5 'terminal cap of globin mRNA to influenza viral complementary RNA during transcription in vitro. Proc. Natl Acad. Sci. USA 76, 1618-1622 (1979).

108. Qi, X. et al. Cap binding and immune evasion revealed by Lassa nucleoprotein structure. Nature $\mathbf{4 6 8}$ 779-783 (2010).

109. Bishop, D. H. M. Y. Gay, M. E. \& Matsuoko, Y. Non Viral heterogeneous sequences are present at the $5^{\prime}$ ends of one species of snowshoe hare bunyavirus $\mathrm{S}$. complementary RNA. Nucleic Acids Res. 11 6409-6418 (1983)

110. Bouloy, M., Pardigon, N., Vialat, P., Gerbaud, S. \& Girard, M. Characterization of the $5^{\prime}$ and $3^{\prime}$ ends of viral messenger RNAs isolated from BHK21 cells infected with Germiston virus (Bunyavirus). Virology 175, 50-58 (1990)

111. Eshita, Y., Ericson, B., Romanowski, V. \& Bishop, D. H. Analyses of the mRNA transcription processes of snowshoe hare bunyavirus S and M RNA species. J. Virol. 55, 681-689 (1985)

112. Patterson, J. L., Holloway, B. \& Kolakofsky, D. La Crosse virions contain a primer-stimulated RNA polymerase and a methylated cap-dependent endonuclease. J. Virol. 52, 215-222 (1984).

113. Garcin, D. et al. The 5' ends of Hantaan virus (Bunyaviridae) RNAs suggest a prime-and-realign mechanism for the initiation of RNA synthesis. J. Virol. 69, 5754-5762 (1995)

114. Jin, H. \& Elliott, R. M. Non-viral sequences at the $5^{\prime}$ ends of Dugbe nairovirus S mRNAs. J. Gen. Virol. 74 2293-2297 (1993).

115. Raju, R et al. Nontemplated bases at the 5'ends of Tacaribe virus mRNAs. Virology 174, 53-59 (1990)

116. Weber, F., Haller, O. \& Kochs, G. Nucleoprotein viral RNA and mRNA of Thogoto virus: a novel" capstealing" mechanism in tick-borne orthomyxoviruses? J. Virol. 70, 8361-8367 (1996).

117. Leahy, M. B., Dessens, J. T. \& Nuttall, P. A. In vitro polymerase activity of Thogoto virus: evidence for unique cap-snatching mechanism in a tick-borne orthomyxovirus. J. Virol. 71, 8347-8351 (1997).

118. Guilligay, D. et al. The structural basis for cap binding by influenza virus polymerase subunit PB2. Nature Struct. Mol. Biol. 15, 500-506 (2008).

119. Dias, A. et al. The cap-snatching endonuclease of influenza virus polymerase resides in the PA subunit. Nature 458, 914-918 (2009).

120. Yuan, P. et al. Crystal structure of an avian influenza polymerase $\mathrm{PA}_{\mathrm{N}}$ reveals an endonuclease active site. Nature 458, 909-913 (2009).

Together with reference 119 , this paper describes the structural and functional characterization of the cap-snatching endonuclease.

121. Crépin, T. et al. Mutational and metal binding analysis of the endonuclease domain of the influenza virus polymerase PA subunit. J. Virol. 84, 9096-9104 (2010).

122. Reguera, J., Weber, F. \& Cusack, S. Bunyaviridae RNA polymerases (L-protein) have an $\mathrm{N}$-terminal, influenzalike endonuclease domain, essential for viral capdependent transcription. PLoS Pathog. 6, e 1001101 (2010)

123. Morin, B. et al. The N-terminal domain of the arenavirus $L$ protein is an RNA endonuclease essentia in mRNA transcription. PLoS Pathog. 6, e1001038 (2010)

124. Lelke, M., Brunotte, L., Busch, C. \& Günther, S. An $\mathrm{N}$-terminal region of Lassa virus $\mathrm{L}$ protein plays a critical role in transcription but not replication of the virus genome. J. Virol. 84, 1934-1944 (2010)

125. Kranzusch, P. J. et al. Assembly of a functional Machupo virus polymerase complex. Proc. Natl Acad. Sci. USA 107, 20069-20074 (2010).

126. Ruigrok, R. W. H. Crépin, T., Hart, D. J. \& Cusack, S. Towards an atomic resolution understanding of the influenza virus replication machinery. Curr. Opin. Struct. Biol. 20, 104-113 (2010).

127. Hastie, K. M., Kimberlin, C. R., Zandonatti, M. A., MacRae, I. J. \& Saphire, E. O. Structure of the Lassa virus nucleoprotein reveals a dsRNA-specific $3^{\prime}$ to $5^{\prime}$ exonuclease activity essential for immune suppression. Proc. Natl Acad. Sci. USA 108, 2396-2401 (2011).

128. Mir, M. A., Duran, W. A., Hjelle, B. L., Ye, C. $\delta$ Panganiban, A. T. Storage of cellular $5^{\prime}$ mRNA caps in P bodies for viral cap-snatching. Proc. Natl Acad. Sci. USA 105, 19294-19299 (2008).

An article that casts light on how $\mathrm{N}$ protein binds preferentially to capped mRNAs, stores and protects these mRNAs in P-bodies, and potentially takes an active role in cap acquisition.

129. Ferron, F. et al. The hexamer structure of the rift valley fever virus nucleoprotein suggests a mechanism for its assembly into ribonucleoprotein complexes. PLOS Pathog. 7, e1002030 (2011)

130. Koyama, S., Ishii, K. J., Coban, C. \& Akira, S. Innate immune response to viral infection. Cytokine 43, 336-341 (2008)

131. Takeuchi, O. \& Akira, S. Recognition of viruses by innate immunity. Immunol. Rev. 220, 214-224, (2007).

132. Wilkins, C. \& Gale, M., Jr Recognition of viruses by cytoplasmic sensors. Curr. Opin. Immunol. 22, 41-47 (2010)

133. Yoneyama, M. \& Fujita, T. Recognition of viral nucleic acids in innate immunity. Rev. Med. Virol. 20, 4-22 (2010)

134. Brennan, K. \& Bowie, A. G. Activation of host pattern recognition receptors by viruses. Curr. Opin. Microbiol. 13, 503-507 (2010)

135. Hansen, J. D., Vojtech, L. N. \& Laing, K. J. Sensing disease and danger: a survey of vertebrate PRRs and their origins. Dev. Comp. Immunol. 35, 886-897 (2011)

136. Meylan, E., Tschopp, J. \& Karin, M. Intracellular pattern recognition receptors in the host response. Nature 442, 39-44 (2006).

137. Diebold, S. S., Kaisho, T., Hemmi, H., Akira, S. \& Reis e Sousa, C. Innate antiviral responses by means of TLR7-mediated recognition of single-stranded RNA. Science 303, 1529-1531 (2004).

138. Züst, R. et al. Ribose 2'-O-methylation provides a molecular signature for the distinction of self and nonself mRNA dependent on the RNA sensor Mda5. Nature Immunol. 12, 137-143 (2011). The authors show for the first time the role of 2'-O-methylation in the sensing of self RNA by the innate immune system through the RNA sensor MDA5.

139 Oshiumi, H. Sakai, K., Matsumoto, M. \& Seya, T DEAD/H BOX 3 (DDX3) helicase binds the RIG-I adaptor IPS- 1 to up-regulate IFN- $\beta$-inducing potential. Eur. J. Immunol. 40, 940-948 (2010)

140. Pichlmair, A. et al. RIG-I-mediated antiviral responses to single-stranded RNA bearing 5'-phosphates. Science 314, 997-1001 (2006). The 5'-triphosphate of viral RNA is identified as a major component in the RIG-I-mediated host innate immune response.

141. Schmidt, A. et al. 5'-triphosphate RNA requires basepaired structures to activate antiviral signaling via RIG-I. Proc. Natl Acad. Sci. USA 106, 12067-12072 (2009).

142. Wang, Y. et al. Structural and functional insights into 5'-ppp RNA pattern recognition by the innate immune receptor RIG-I. Nature Struct. Mol. Biol. 17, 781-787 (2010)

143. Luthra, P., Sun, D., Silverman, R. H. \& He, B. Activation of IFN $-\beta$ expression by a viral mRNA through RNase L and MDA5. Proc. Natl Acad. Sci. USA 108, 2118-2123 (2011).

144. Li, X. et al. Structural basis of double-stranded RNA recognition by the RIG-I like receptor MDA5. Arch. Biochem. Biophys. 488, 23-33 (2009).

145. Daffis, S. et al. 2'-O methylation of the viral mRNA cap evades host restriction by IFIT family members. Nature 468, 452-456 (2010). The authors demonstrate that 2'-O-methylation of the viral RNA cap evades host innate antiviral responses through escape of IFIT-mediated suppression. 
146. Pichlmair, A. et al. IFIT1 is an antiviral protein that recognizes 5'-triphosphate RNA. Nature Immunol. 12, 624-630 (2011)

147. Garcin, D. \& Kolakofsky, D. A novel mechanism for the initiation of Tacaribe arenavirus genome replication. J. Virol. 64, 6196-6203 (1990).

148. Hong, Z. \& Cameron, C. E. Pleiotropic mechanisms of ribavirin antiviral activities. Prog. Drug Res. 59, 41-69 (2002)

149. Magden, J., Kääriäinen, L. \& Ahola, T. Inhibitors of virus replication: recent developments and prospects. Appl. Microbiol. Biotechnol. 66, 612-621 (2005).

150. Lim, S. P. et al. Small molecule inhibitors that selectively block dengue virus methyltransferase. J. Biol. Chem. 286, 6233-6240 (2011).

151. Kuzuhara, T., Iwai, Y., Takahashi, H., Hatakeyama, D. \& Echigo, N. Green tea catechins inhibit the endonuclease activity of influenza A virus RNA polymerase. PLoS Curr. 1, RRN 1052 (2009).

152. Parkes, K. E. B. et al. Use of a pharmacophore model to discover a new class of influenza endonuclease inhibitors. J. Med. Chem. 46, 1153-1164 (2003).

153. Tomassini, J. et al. Inhibition of cap ( $\left.\mathrm{m}^{7} \mathrm{GpppXm}\right)$ dependent endonuclease of influenza virus by 4-substituted 2,4-dioxobutanoic acid compounds. Antimicrob. Agents Chemother. 38, 2827-2837 (1994).

154. Balvay, L., Soto Rifo, R., Ricci, E. P., Decimo, D. \& Ohlmann, T. Structural and functional diversity of viral IRESes. Biochim. Biophys. Acta 1789, 542-557 (2009).
155. Guidotti, L. G. \& Chisari, F. V. Noncytolytic control of viral infections by the innate and adaptive immune response. Annu. Rev. Immunol. 19, 65-91 (2001).

156. Malmgaard, L. Induction and regulation of IFNs during viral infections. J. Interferon Cytokine Res. 24 439-454 (2004)

157. Garaigorta, U. \& Chisari, F. V. Hepatitis C virus blocks interferon effector function by inducing protein kinase R phosphorylation. Cell Host Microbe 6 , 513-522 (2009).

158. Blanc, A., Goyer, C. \& Sonenberg, N. The coat protein of the yeast double-stranded RNA virus L-A attaches covalently to the cap structure of eukaryotic mRNA. Mol. Cell. Biol. 12, 3390-3398 (1992).

159. Naitow, H., Tang, J., Canady, M., Wickner, R. B. \& Johnson, J. E. L-A virus at 3.4 A resolution reveals particle architecture and mRNA decapping mechanism. Nature Struct. Biol. 9, 725-728 (2002).

160. Parrish, S., Resch, W. \& Moss, B. Vaccinia virus D10 protein has mRNA decapping activity, providing a mechanism for control of host and viral gene expression. Proc. Natl Acad. Sci. USA 104, 2139-2144 (2007)

161. McLennan, A. G. Decapitation: poxvirus makes RNA lose its head. Trends Biochem. Sci. 32, 297-299 (2007).

162. Gaglia, M. M. \& Glaunsinger, B. A. Viruses and the cellular RNA decay machinery. Wiley Interdiscip. Rev. RNA 1, 47-59 (2010).

\section{Acknowledgements}

The authors are in debt to all previous and current laboratory members, too numerous to name, for their contributions and involvement in the study of viral RNA replication and capping. Special thanks go to B. Selisko for her tireless dedication to and help with the scientific elaboration of this manuscript. This work was supported in part through funding by the Fondation pour la Recherche Médicale (Programme Aide aux équipes), the French Direction Gênérale de l'Armement (contrat 07co404), Infectiopôle-Sud and the European Union Seventh Framework Programme (FP7/2007-2013) through the project SILVER (Small inhibitor leads against emerging RNA viruses; grant agreement 260644).

Competing interests statement

The authors declare no competing financial interests.

\section{DATABASES}

Protein Data Bank: http://www.cancer.gov/drugdictionary 1 AV6 $|\underline{1 C K M}| \underline{1 C K N}|\underline{1 C K O}| \underline{1 E} 6|\underline{1 L 9 V}| \underline{1 Y N 9}|\underline{2 B H R}|$ 2JHC|2P41|2QZE |2VDW |2VOZ |2XI5 |3HW4|3JSB

FURTHER INFORMATION

\section{Bruno Canard's homepage:}

http://www.afmb.univ-mrs.fr/Bruno-Canard

PyMOL: http://www.pymol.org

ALL LINKS ARE ACTIVE IN THE ONLINE PDF 